\title{
A Versatile Method for the Synthesis of 3-Alkoxycarbonyl $\beta$-Lactam Derivatives
}

\author{
Lei Jiao, Qianfeng Zhang, Yong Liang, Shiwei Zhang, and Jiaxi Xu* \\ Key Laboratory of Bioorganic Chemistry and Molecular Engineering of Ministry of Education, College of \\ Chemistry and Molecular Engineering, Peking University, Beijing 100871, P. R. China
}

\section{Supplemental Materials}

Content S1

Experimental Details. $\mathrm{S} 2$

Additional Discussion on the trans Selectivity of the Desulfurization Reactions S3

The Spectroscopic Data for the Products .... S5

Copies of ${ }^{1} \mathrm{H}$ NMR Spectra of the Products. S9

Crystal Structure of Polycyclic $\beta$-Lactam 4i (ORTEP Figure) S27 


\section{Experimental Details}

General. Diazo thioester $\mathbf{1}^{1}$, imines $3 \mathbf{a}-\mathbf{f}^{2}, \mathbf{3 g}-\mathbf{h}^{3}$, and $\mathbf{3 i}{ }^{4}$ were prepared according to published procedures. Rhodium(II) acetate, tributylstannane, AIBN, zinc powder, and glacial acetic acid were commercial reagents and were used as received. Toluene was refluxed with sodium and benzophenone, and freshly distilled prior to use. All reactions were performed under a nitrogen atmosphere. Melting points were measured on a melting point apparatus and are uncorrected. ${ }^{1} \mathrm{H}$ NMR (300 or $400 \mathrm{MHz}$ ) and ${ }^{13} \mathrm{C}$ NMR (75 or $100 \mathrm{MHz}$ ) spectra were recorded in $\mathrm{CDCl}_{3}$ with TMS as an internal standard. Mass spectra and HRMS data were obtained on an EI mass spectrometer. IR spectra were recorded on a FT-IR spectrometer with an OMNI sampler.

The Unsuccessful Acyl Chloride-Imine Reaction. The reaction of ethyl malonyl chloride with $N$-benzylideneaniline in the presence of triethylamine was conducted according to the well-established acyl chloride-imine method: ${ }^{5}$ To a solution of $N$-benzylideneaniline $(86 \mathrm{mg}, 0.48 \mathrm{mmol})$ and triethylamine ( $71 \mathrm{mg}, 0.70 \mathrm{mmol})$ in $10 \mathrm{~mL}$ of dry toluene at ambient temperature was added a solution of ethyl malonyl chloride (100 mg, $0.66 \mathrm{mmol})$ in $5 \mathrm{~mL}$ of dry toluene during a period of $30 \mathrm{~min}$. The resulting mixture was stirred at ambient temperature for another $1 \mathrm{~h}$ and was monitored by thin layer chromatography (silica gel $\mathrm{GF}_{254}$ plate, hexanes / ethyl acetate 5:1 V/V). It was found that, although the $N$-benzylideneaniline was completely converted, no desired 3-ethoxycarbonyl $\beta$-lactam derivative (5a or 5b) was found (compared with the standard sample of $\mathbf{5 a}$ and $\mathbf{5 b}$ prepared by our methods) and high-polar impurities were formed. This indicates that the acyl chloride-imine method cannot approach the synthetic goal towards the 3-ethoxycarbonyl $\beta$-lactam derivatives.

\footnotetext{
${ }^{1}$ Marino, J. P. Jr.; Osterhout, M. H.; Price, A. T.; Sheehan, S. M.; Padwa, A. Tetrahedron Lett. 1994, 35, 849-852.

2 Linder, M. R.; Frey, W. U.; Podlech, J. J. Chem. Soc., Perkin Trans. 1 2001, 2566-2577.

Wenker, H. J. Am. Chem. Soc. 1935, 57, 6, 1079-1080.

${ }_{5}^{4}$ Xu, J. X.; Jin S.; Xing, Q. Y. Phosphorus, Sulfur and Silicon 1998, 141, 57-70.

5 The Organic Chemistry of $\beta$-Lactams; Georg, G. I., Ed.; Verlag Chemie: New York, 1993.
} 


\section{Additional Discussion on the trans Selectivity of the Desulfurization Reactions}

Cis-3,4-disubstituted $\beta$-lactams were generally obtained as major products in Raney $\mathrm{Ni}$ and $n-\mathrm{Bu}_{3} \mathrm{SnH} / \mathrm{AIBN}$ promoted desulfurizations due to the hydrogen addition to the less hindered face of the radical intermediate. ${ }^{6}$ It is noteworthy that the relative configurations of the predominating 3 -ethoxycarbonyl $\beta$-lactam products are thermodynamically more stable trans isomeric in our cases, which is quite different from those previously reported. ${ }^{6}$ Because of the presence of the 3-ethoxycarbonyl group in the substrates, the products possess enolizable centers. Thus, both desulfurization methods lead to the thermodynamically more stable isomers, the trans-3,4-disubstituted $\beta$-lactams, via a tautomeric equilibrium of the two diastereomers through their enolic intermediates under the reaction conditions.

A series of experiments were conducted (Scheme 1) to verify this proposal.

\section{Scheme 1}

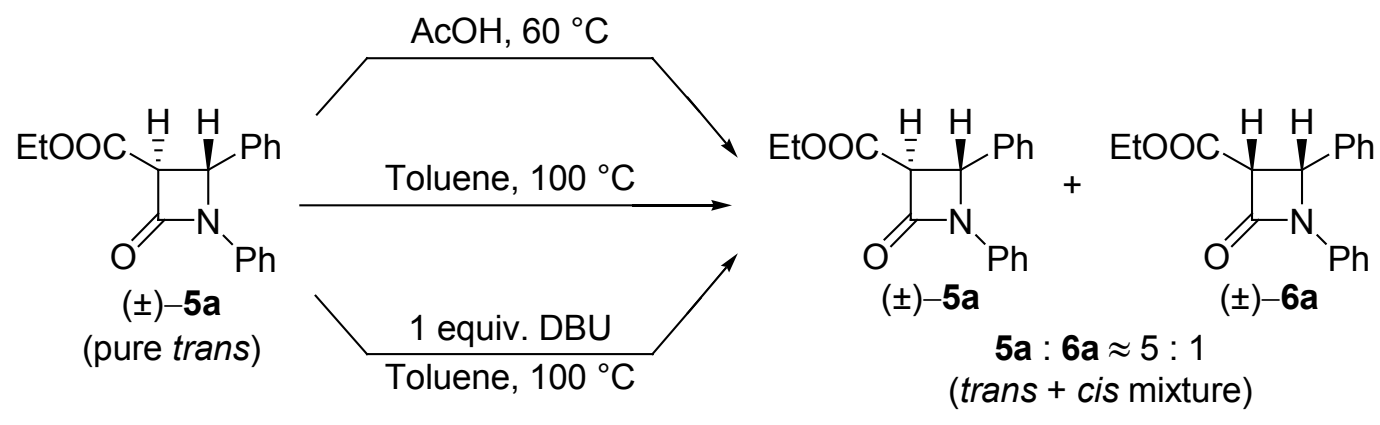

Pure trans $-\beta$-lactam 5a was obtained by recrystallization (from ethanol / hexanes) of the desulfurization products $(\mathbf{5 a}+\mathbf{5 b})$, suggesting that the tautomerization does not occur at room temperature. Then pure $\mathbf{5 a}$ (13 mg each, $0.044 \mathrm{mmol}$ ) was solved in $1 \mathrm{~mL}$ of glacial acetic acid, in $1 \mathrm{~mL}$ of dry toluene, and in $1 \mathrm{~mL}$ of dry toluene plus $6 \mu \mathrm{L}$ of DBU (1 equiv.), ${ }^{7}$ respectively. The first solution was maintained at $60{ }^{\circ} \mathrm{C}$, and the other two were maintained at $100{ }^{\circ} \mathrm{C}$ for $2 \mathrm{~h}$ to simulate the desulfurization reaction conditions. After the removal of the solvent, the residue was submitted to NMR analysis. ${ }^{1} \mathrm{H}$ NMR results showed that pure $\mathbf{5 a}$ tautomerized to a mixture of $5 \mathbf{a}$ and $\mathbf{6 a}$ after the above treatments, and the 5a / 6a ratios were about 5:1, which was very near to that of the product mixtures of desulfurization reactions (5a / 6a equals to 88: 12 for $n-\mathrm{Bu}_{3} \mathrm{SnH} / \mathrm{AIBN}$ method and $84: 16$ for $\mathrm{Zn} / \mathrm{AcOH}$ method). This indicates that, the trans selectivity of the desulfurization reactions is due to the tautomeric equilibrium of the two diastereomers under the reaction conditions, and the products (5a and $\mathbf{6 a})$ are thermodynamic mixture of isomers.

Another experiment was also conducted: to a solution of product mixture $\mathbf{5 i}$ and $\mathbf{6 i}(250 \mathrm{mg}, 0.58 \mathrm{mmol}$, $5 \mathbf{i} / \mathbf{6 i}=67: 33)$ in $20 \mathrm{~mL}$ of dry benzene was added $80 \mu \mathrm{L}$ of DBU (1 equiv.), ${ }^{6}$ and the solution was

\footnotetext{
${ }^{6}$ (a) Bose, A. K.; Manhas, M. S.; Chib, J. B.; Chawla, H. P. S.; Dayal, B. J. Org. Chem. 1974, 39, 2877-2884. (b) Bateson, J. H.; Quinn, A. M.; Southgate, R. J. Chem. Soc., Chem. Commun. 1986, 1151-1152. (c) Palomo, C.; Cossio, F. P.; Odriozola, J. M.; Oiarbide, M.; Ontoria, J. M. Tetrahedron Lett. 1989, 30, 4577-4580. (d) Palomo, C.; Cossio, F. P.; Odriozola, J. M.; Oiarbide, M.; Ontoria, J. M. J. Org. Chem. 1991, 56, 4418-4428. (e) Bari, S. S.; Venugopalan, P.; Arora, R. Tetrahedron Lett. 2003, 44, 895-897.

7 The use of DBU or DBN is a method that can isomerize the 3-acetyl cis $\beta$-lactams to the more stable trans forms. See: Manhas, M. S.; Ghosh, M.; Bose, A. K. J. Org. Chem. 1990, 55, 575-580.
} 
refluxed overnight. The resulting mixture was successively washed with $1 \mathrm{~mol} / \mathrm{L} \mathrm{HCl}$, saturated $\mathrm{NaHCO}_{3}$, and brine, and then dried over $\mathrm{Na}_{2} \mathrm{SO}_{4}$. After the evaporation of the solvent, the residue was submitted to NMR analysis, and it was found that the product ratio $\mathbf{5 i}$ / 6i maintained at 67: 33. This also suggests that the mixture of $\mathbf{5 i}$ and $\mathbf{6} \mathbf{i}$ is a thermodynamic mixture, and the observed trans selectivity should be attributed to the tautomeric equilibrium of the two diastereomers under the reaction conditions. 


\section{The Spectroscopic Data for the Products}

Ethyl ( \pm )-trans-2-oxo-1,4-diphenyl-3-phenylthioazetidine-3-carboxylate (4a)

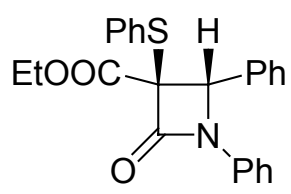

Colorless crystals, m.p. $93-94{ }^{\circ} \mathrm{C} .{ }^{1} \mathrm{H}$ NMR (300 MHz): $\delta 0.78(\mathrm{t}, J=7.2 \mathrm{~Hz}, 3 \mathrm{H}), 3.68$ (q, $\left.J=7.2 \mathrm{~Hz}, 2 \mathrm{H}\right)$, $5.06(\mathrm{~s}, 1 \mathrm{H}), 7.06-7.32(\mathrm{~m}, 13 \mathrm{H}), 7.79(\mathrm{~m}, 2 \mathrm{H}) .{ }^{13} \mathrm{C}$ NMR (75.5 MHz): $\delta$ 13.4, 62.0, 65.0, 71.2, 117.2, 124.4, 126.9, 128.6, 128.95, 128.99, 129.1, 129.4, 132.5, 135.3, 136.7, 161.0, 164.9. MS (EI) m/z: $403\left(\mathrm{M}^{+}\right.$, 4.9), 284 (86), 181 (100). IR $v\left(\mathrm{~cm}^{-1}\right)$ : 1736, 1758. Calcd for $\mathrm{C}_{24} \mathrm{H}_{21} \mathrm{NO}_{3} \mathrm{~S}: \mathrm{C}, 71.44 ; \mathrm{H}, 5.25 ; \mathrm{N}, 3.47$. Found: C, 71.47; H, 5.21; N, 3.33 .

Ethyl ( \pm )-trans $-1-(4-$ methoxyphenyl $)-2-0 x 0^{-3}-$ phenylthio-4-styrylazetidine-3-carboxylate (4b)<smiles>CCOC(=O)C1(S)C(=O)N(P)C1/C=C/c1ccccc1</smiles>

Colorless oil. ${ }^{1} \mathrm{H}$ NMR (300 MHz): $\delta 1.10$ (t, $\left.J=7.2 \mathrm{~Hz}, 3 \mathrm{H}\right), 3.74$ (s, 3H), 4.14 (dq, $J=1.8,7.2 \mathrm{~Hz}, 2 \mathrm{H}$ ), $4.61(\mathrm{dd}, J=0.3,7.5 \mathrm{~Hz}, 1 \mathrm{H}), 6.25(\mathrm{dd}, J=7.8,15.9 \mathrm{~Hz}, 1 \mathrm{H}), 6.80(\mathrm{~m}, 3 \mathrm{H}), 7.26-7.35(\mathrm{~m}, 10 \mathrm{H}), 7.76(\mathrm{~m}$, $2 \mathrm{H}) .{ }^{13} \mathrm{C}$ NMR (75.5 MHz): $\delta 14.0,55.3,62.4,63.8,70.6,114.2,118.6,121.6,126.7,128.7,129.0,129.1$, 129.3, 130.5, 135.1, 135.2, 136.8, 156.4, 160.0, 165.5. MS (EI) m/z: $459\left(\mathrm{M}^{+}, 2.3\right), 310$ (4.2), 236 (100). IR $v\left(\mathrm{~cm}^{-1}\right): 1735,1761$. Calcd for $\mathrm{C}_{27} \mathrm{H}_{25} \mathrm{NO}_{4} \mathrm{~S}: 459.1504$. Found: 459.1511.

Ethyl ( \pm )-trans-1-benzyl-2-oxo-4-phenyl-3-phenylthioazetidine-3-carboxylate (4d)<smiles>CCOC(=O)C1(c2ccccc2)C(=O)N(Cc2ccccc2)C1c1ccccc1</smiles>

Colorless crystals, m.p. $161-162{ }^{\circ} \mathrm{C} .{ }^{1} \mathrm{H}$ NMR (300 MHz): $\delta 0.80$ (t, $\left.J=7.2 \mathrm{~Hz}, 3 \mathrm{H}\right), 3.71$ (q, $J=7.2 \mathrm{~Hz}$, 2H), $3.86(\mathrm{~d}, J=15.0 \mathrm{~Hz}, 1 \mathrm{H}), 4.46(\mathrm{~s}, 1 \mathrm{H}), 4.86(\mathrm{~d}, J=15.0 \mathrm{~Hz}, 1 \mathrm{H}), 6.77-7.75(\mathrm{~m}, 15 \mathrm{H}) .{ }^{13} \mathrm{C}$ NMR $(75.5$ MHz): $\delta 13.4,44.6,61.9,64.6,72.6,127.1,127.6,128.1,128.5,128.6,128.8,129.0,129.1,129.4,132.6$, 133.8, 135.8, 163.6, 165.2. MS (EI) m/z: $417\left(\mathrm{M}^{+}, 1.0\right), 284$ (100), 195 (12), 91 (82). IR $v\left(\mathrm{~cm}^{-1}\right): 1731$, 1772. Calcd for $\mathrm{C}_{25} \mathrm{H}_{23} \mathrm{NO}_{3} \mathrm{~S}: 417.1399$. Found: 417.1410 . 
Ethyl ( $( \pm)-$ trans $^{-1}$-tert-butyl-2-oxo-4-phenyl-3-phenylthioazetidine-3-carboxylate (4e)<smiles>CCOC(=O)[C@](S)(c1ccccc1)[C@H](c1ccccc1)C(C)(C)C</smiles>

Colorless crystals, m.p. $75-76{ }^{\circ} \mathrm{C} .{ }^{1} \mathrm{H}$ NMR (300 MHz): $\delta 1.08$ (t, $\left.J=7.2 \mathrm{~Hz}, 3 \mathrm{H}\right), 1.33$ (s, 9H), 4.12 (m, 2H), $5.23(\mathrm{~s}, 1 \mathrm{H}), 7.10-7.38(\mathrm{~m}, 10 \mathrm{H}) .{ }^{13} \mathrm{C}$ NMR (75.5 MHz): $\delta$ 13.7, 28.0, 55.3, 62.4, 62.9, 69.4, 127.8, 128.0, 128.4, 128.7, 129.0, 130.6, 133.0 135.2, 163.2, 167.4. MS (EI) m/z: $383\left(\mathrm{M}^{+}, 0.1\right), 284$ (100), 211 (86), 135 (77). IR $v\left(\mathrm{~cm}^{-1}\right)$ : 1720, 1762. Calcd for $\mathrm{C}_{22} \mathrm{H}_{23} \mathrm{NO}_{5} \mathrm{~S}$ : 383.1555 . Found: 383.1558.

Ethyl ( \pm )-trans $-1-(2-$ ethoxy-2-oxoethyl $)-2-$ oxo-4-phenyl-3-phenylthioazetidine-3-carboxylate (4f)<smiles>CCOC(=O)CN1C(=O)C(c2ccccc2)(c2ccccc2)C(OCC)C1(O)c1ccccc1</smiles>

Colorless oil. ${ }^{1} \mathrm{H}$ NMR (300 MHz): $\delta 0.75(\mathrm{t}, J=7.2 \mathrm{~Hz}, 3 \mathrm{H}), 1.24(\mathrm{t}, J=7.2 \mathrm{~Hz}, 3 \mathrm{H}), 3.56(\mathrm{~d}, J=18.0 \mathrm{~Hz}$, $1 \mathrm{H}), 3.64(\mathrm{q}, J=7.2 \mathrm{~Hz}, 2 \mathrm{H}), 4.14(\mathrm{~m}, 2 \mathrm{H}), 4.42(\mathrm{~d}, J=18.0 \mathrm{~Hz}, 1 \mathrm{H}), 5.03(\mathrm{~s}, 1 \mathrm{H}), 7.26-7.38(\mathrm{~m}, 8 \mathrm{H})$, 7.78 (m, 2H). ${ }^{13} \mathrm{C}$ NMR (75.5 MHz): $\delta 13.2,13.8,41.4,61.4,61.6,65.62,65.64,72.2,126.9,128.5,128.8$, 128.9, 129.1, 129.6, 132.5, 134.7, 164.1, 164.9, 167.0. MS (EI) m/z: 413 (M+, 2.2), 284 (100), 222 (57). IR $v\left(\mathrm{~cm}^{-1}\right): 1742,1781$. Calcd for $\mathrm{C}_{22} \mathrm{H}_{23} \mathrm{NO}_{5} \mathrm{~S}: 413.1297$. Found: 413.1301 .

Ethyl ( \pm )-trans $-8-0 \times 0-6$-phenyl-7-phenylthio-5-thia-1-aza-bicyclo[4.2.0]octane-7-carboxylate (4h)<smiles>CCOC(=O)C1(S)C(=O)N2CCCSC21c1ccccc1</smiles>

Colorless crystals, m.p. $105-106{ }^{\circ} \mathrm{C} .{ }^{1} \mathrm{H}$ NMR $(300 \mathrm{MHz}): \delta 1.13(\mathrm{t}, J=7.2 \mathrm{~Hz}, 3 \mathrm{H}), 1.86(\mathrm{~m}, 2 \mathrm{H}), 2.65(\mathrm{~m}$, $2 \mathrm{H}), 3.17(\mathrm{~m}, 1 \mathrm{H}), 4.12(\mathrm{~m}, 3 \mathrm{H}), 7.19-7.28(\mathrm{~m}, 3 \mathrm{H}), 7.40-7.47(\mathrm{~m}, 5 \mathrm{H}), 7.63(\mathrm{~m}, 2 \mathrm{H}) .{ }^{13} \mathrm{C}$ NMR $(75.5$ MHz): $\delta 13.9,23.7,25.8,37.9,62.0,70.6,75.7,128.1,128.6,129.0,129.1,135.5,136.2,163.8,164.9$. MS (EI) m/z: $399\left(\mathrm{M}^{+}, 4.1\right), 290$ (1.6), 222 (57), 150 (100). IR $v\left(\mathrm{~cm}^{-1}\right)$ : 1732, 1780. Calcd for $\mathrm{C}_{21} \mathrm{H}_{21} \mathrm{NO}_{3} \mathrm{~S}_{2}: \mathrm{C}$, 63.13; H, 5.30; N, 3.51. Found: C, 63.17; H, 5.21; N, 3.40. 
Ethyl $r e l-(2 S, 2 \mathrm{a} R, 4 R)-1-0 x 0^{-2 a}, 4-d i p h e n y l-2-p h e n y l t h i o-2,2 a, 3,4-t e t r a h y d r o-1 H-a z e t o[2,1-d]$ $[1,5]$ benzothiazepine-2-carboxylate (4i)

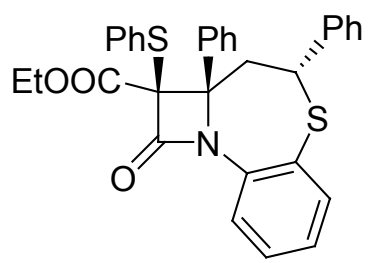

Colorless crystals, m.p. $176-177^{\circ} \mathrm{C} .{ }^{1} \mathrm{H}$ NMR $(300 \mathrm{MHz}): \delta 1.08$ (t, $\left.J=7.2 \mathrm{~Hz}, 3 \mathrm{H}\right), 2.94$ (dd, $J=11.4$, $13.8 \mathrm{~Hz}, 1 \mathrm{H}), 3.25$ (d, $J=13.8 \mathrm{~Hz}, 1 \mathrm{H}), 3.90(\mathrm{~d}, J=11.4 \mathrm{~Hz}, 1 \mathrm{H}), 4.10(\mathrm{~m}, 2 \mathrm{H}), 7.13-7.45(\mathrm{~m}, 16 \mathrm{H}), 7.66$ (m, 2H), 8.00 (d, $J=7.8 \mathrm{~Hz}, 1 \mathrm{H}) .{ }^{13} \mathrm{C}$ NMR (75.5 MHz): $\delta 13.8,45.2,49.1,62.2,71.4,74.0,126.7,126.9$, $128.0,128.2,128.4,128.5,128.6,128.7,128.8,129.0,129.9,130.0,132.1,134.8,135.5,137.7,140.5$, 162.9, 166.3. MS (EI) m/z: 537 (0.9), 491 (48), 314 (42), 211 (100). IR $v\left(\mathrm{~cm}^{-1}\right): 1724,1770$. Calcd for $\mathrm{C}_{32} \mathrm{H}_{27} \mathrm{NO}_{3} \mathrm{~S}_{2}$ : C, 71.48; H, 5.06; N, 2.60. Found: C, 71.49; H, 5.05; N, 2.45.

\section{Ethyl ( \pm )-trans $-2-0 x 0-1,4-$ diphenylazetidine-3-carboxylate (5a)}<smiles>CCOC(=O)C1C(=O)N(c2ccccc2)C1c1ccccc1</smiles>

Colorless crystals, m.p. $87-89^{\circ} \mathrm{C}$. Lit. ${ }^{8}$ m.p. $87-89^{\circ} \mathrm{C}$ The spectroscopic data is identical to the literature. ${ }^{8}$

Ethyl ( \pm )-trans-1-(4-methoxyphenyl)-2-oxo-4-styrylazetidine-3-carboxylate (5b)<smiles>CCOC(=O)C1C(=O)N([Pb])C1/C=C/c1ccccc1</smiles>

Colorless oil. ${ }^{1} \mathrm{H}$ NMR (300 MHz): $\delta 1.32(\mathrm{t}, J=7.2 \mathrm{~Hz}, 3 \mathrm{H}), 3.75(\mathrm{~s}, 3 \mathrm{H}), 3.96(\mathrm{~d}, J=2.4 \mathrm{~Hz}, 1 \mathrm{H}), 4.28$ (q, $J=7.2 \mathrm{~Hz}, 2 \mathrm{H}), 4.94(\mathrm{dd}, J=2.4,8.4 \mathrm{~Hz}, 1 \mathrm{H}), 6.26(\mathrm{dd}, J=8.4,15.9 \mathrm{~Hz}, 1 \mathrm{H}), 6.86(\mathrm{~m}, 3 \mathrm{H}), 7.26-7.41$ (m, $7 \mathrm{H}) .{ }^{13} \mathrm{C}$ NMR (75.5 MHz): $\delta$ 14.1, 55.4, 56.8, 60.9, 62.0, 114.3, 118.4, 125.1, 126.6, 128.6, 128.7, 131.1, 135.2, 135.5, 156.3, 158.3, 166.4. MS (EI) m/z: $351\left(\mathrm{M}^{+}, 10\right), 278$ (13), 236 (10), 202 (31), 149 (100), 129 (72). IR $v\left(\mathrm{~cm}^{-1}\right): 1728,1758$. Calcd for $\mathrm{C}_{21} \mathrm{H}_{21} \mathrm{NO}_{4}: 351.1471$. Found: 351.1469 .

\section{Ethyl ( \pm )-trans $-1-$-benzyl-2-oxo-4-phenylazetidine-3-carboxylate (5d)}<smiles>CCOC(=O)C1C(=O)N(Cc2ccccc2)C1c1ccccc1</smiles>

Colorless oil. Known compound. The spectroscopic data is identical to the literature. ${ }^{9}$

\footnotetext{
${ }^{8}$ Brown, D. S.; Elliott, M. C.; Moody, J. C.; Mowlem, T. J.; Marino, J. P.; Padwa, A. J. Org. Chem. 1994, 59, $2447-2455$.
} 
Ethyl ( \pm )-trans $-1-$ tert-butyl-2-oxo-4-phenylazetidine-3-carboxylate (5e)<smiles>CCOC(=O)[C@H]1C(=O)N(C(C)(C)C)[C@H]1c1ccccc1</smiles>

Colorless oil. Known compound, but no spectroscopic data was given in the literature. ${ }^{10}{ }^{1} \mathrm{H}$ NMR (300 MHz): $\delta 1.27$ (s, 9H), 1.29 (t, $J=7.2 \mathrm{~Hz}, 3 \mathrm{H}), 3.70$ (d, $J=2.4 \mathrm{~Hz}, 1 \mathrm{H}), 4.24$ (dq, $J=2.1,7.2 \mathrm{~Hz}, 2 \mathrm{H}), 4.86$ (d, $J=2.4 \mathrm{~Hz}, 1 \mathrm{H}), 7.36-7.43$ (m, 5H). ${ }^{13} \mathrm{C}$ NMR (75.5 MHz): $\delta$ 14.0, 28.0, 55.1, 56.3, 61.6, 62.4, 126.5, 128.6, 128.9, 139.1, 162.0, 167.0. MS (EI) m/z: $275\left(\mathrm{M}^{+}, 3.5\right), 176$ (98), 131 (100). IR $v\left(\mathrm{~cm}^{-1}\right): 1721,1754$. Calcd for $\mathrm{C}_{16} \mathrm{H}_{21} \mathrm{NO}_{3}$ : 275.1521. Found: 275.1524 .

Ethyl ( \pm )-trans-8-0xo-6-phenyl-5-thia-1-aza-bicyclo[4.2.0]octane-7-carboxylate (5h)<smiles>CCOC(=O)C1C(=O)N2CCCS[C@]12c1ccccc1</smiles>

Colorless crystals, m.p. $113-115{ }^{\circ} \mathrm{C} .{ }^{1} \mathrm{H}$ NMR (300 MHz): $\delta 0.82$ (t, $\left.J=7.2 \mathrm{~Hz}, 3 \mathrm{H}\right), 1.86(\mathrm{~m}, 2 \mathrm{H}), 2.66$ (m, 2H), $3.11(\mathrm{~m}, 1 \mathrm{H}), 3.70(\mathrm{q}, J=7.2 \mathrm{~Hz}, 2 \mathrm{H}), 4.18(\mathrm{~m}, 1 \mathrm{H}), 4.38(\mathrm{~s}, 1 \mathrm{H}), 7.33-7.41(\mathrm{~m}, 3 \mathrm{H}), 7.59-7.62(\mathrm{~m}$, 2H). ${ }^{13} \mathrm{C}$ NMR (75.5 MHz): $\delta$ 13.4, 23.4, 25.7, 38.0, 61.2, 70.3, 127.7, 128.1, 128.6, 136.1, 160.9, 164.5 . MS (EI) m/z: $291\left(\mathrm{M}^{+}, 4.0\right), 218$ (100), 130 (29). IR $v\left(\mathrm{~cm}^{-1}\right): 1729,1765$. Calcd for $\mathrm{C}_{15} \mathrm{H}_{17} \mathrm{NO}_{3} \mathrm{~S}: \mathrm{C}, 61.83$; H, 5.88; N, 4.81. Found: C, 61.76; H, 5.86; N, 4.69.

Ethyl rel-(2S,2aS,4R)-1-oxo-2a,4-diphenyl-2,2a,3,4-tetrahydro-1H-azeto[2,1-d][1,5]benzothiazepine-2-carboxylate (5i)<smiles>CCOC(=O)[C@H]1C(=O)N2c3ccccc3S[C@H](c3ccccc3)C[C@]12c1ccccc1</smiles>

Colorless crystals, m.p. $153-155{ }^{\circ} \mathrm{C} .{ }^{1} \mathrm{H}$ NMR (400 MHz): $\delta 0.84$ (t, $\left.J=7.2 \mathrm{~Hz}, 3 \mathrm{H}\right), 3.17$ (dd, $J=11.0$, $14.3 \mathrm{~Hz}, 1 \mathrm{H}), 3.68$ (d, $J=14.3 \mathrm{~Hz}, 1 \mathrm{H}), 3.70$ (q, $J=7.2 \mathrm{~Hz}, 2 \mathrm{H}), 3.97$ (d, $J=11.0 \mathrm{~Hz}, 1 \mathrm{H}), 4.30(\mathrm{~s}, 1 \mathrm{H})$, 7.15-7.42 (m, 13H), $7.95(\mathrm{~m}, 1 \mathrm{H}) .{ }^{13} \mathrm{C}$ NMR (100 MHz): $\delta$ 13.5, 46.1, 50.9, 61.2, 67.6, 76.7, 126.88, $126.91,127.5,128.1,128.3,128.4,129.1,130.2,132.3,136.0,137.9,140.5,160.5,165.3$. MS (EI) m/z: 429 (56), 356 (14), 302 (26), 211 (100). IR $v\left(\mathrm{~cm}^{-1}\right): 1729,1767$. Calcd for $\mathrm{C}_{26} \mathrm{H}_{23} \mathrm{NO}_{3} \mathrm{~S}: 429.1399$. Found: 429.1397.

\footnotetext{
9 Miah, S.; Slawin, A. M. Z.; Moody, C. J.; Sheehan, S. M.; Marino, J. P.; Semones, M. A.; Padwa, A.; Richards, I. C. Tetrahedron 1996, 52, 2489-2514.

${ }^{10}$ Choi, M. K. W.; Yu, W. Y.; Che, C. M. Org. Lett. 2005, 7, 1081-1084.
} 


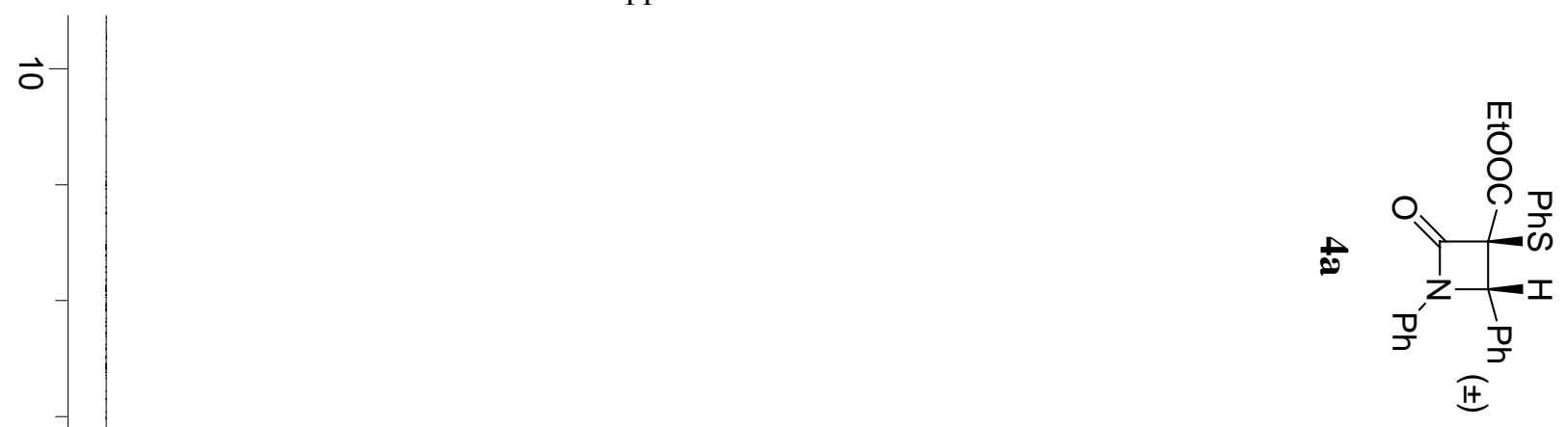

$\infty$

$\overrightarrow{8}$

$\vec{\omega}$

ขु

0

$\dot{8}$

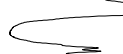

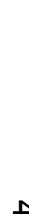

$\triangle$

N

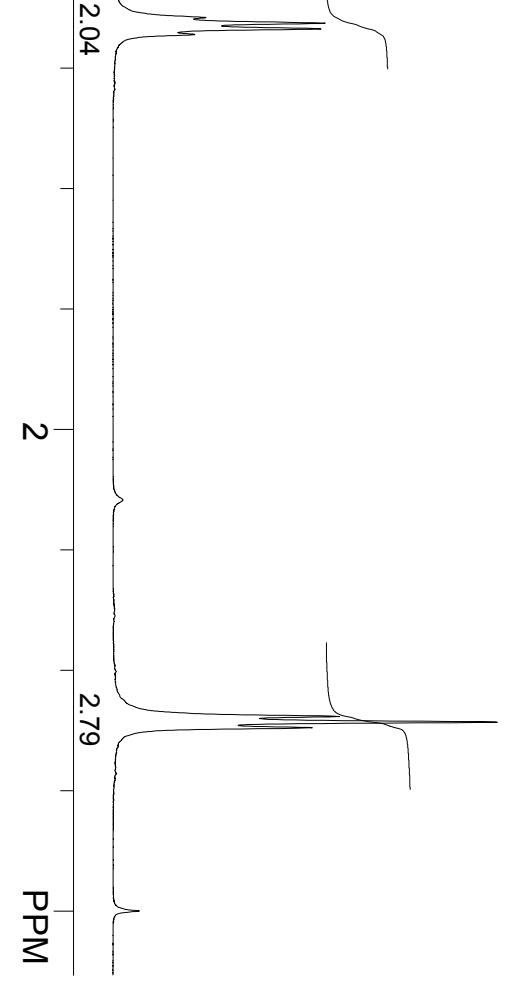




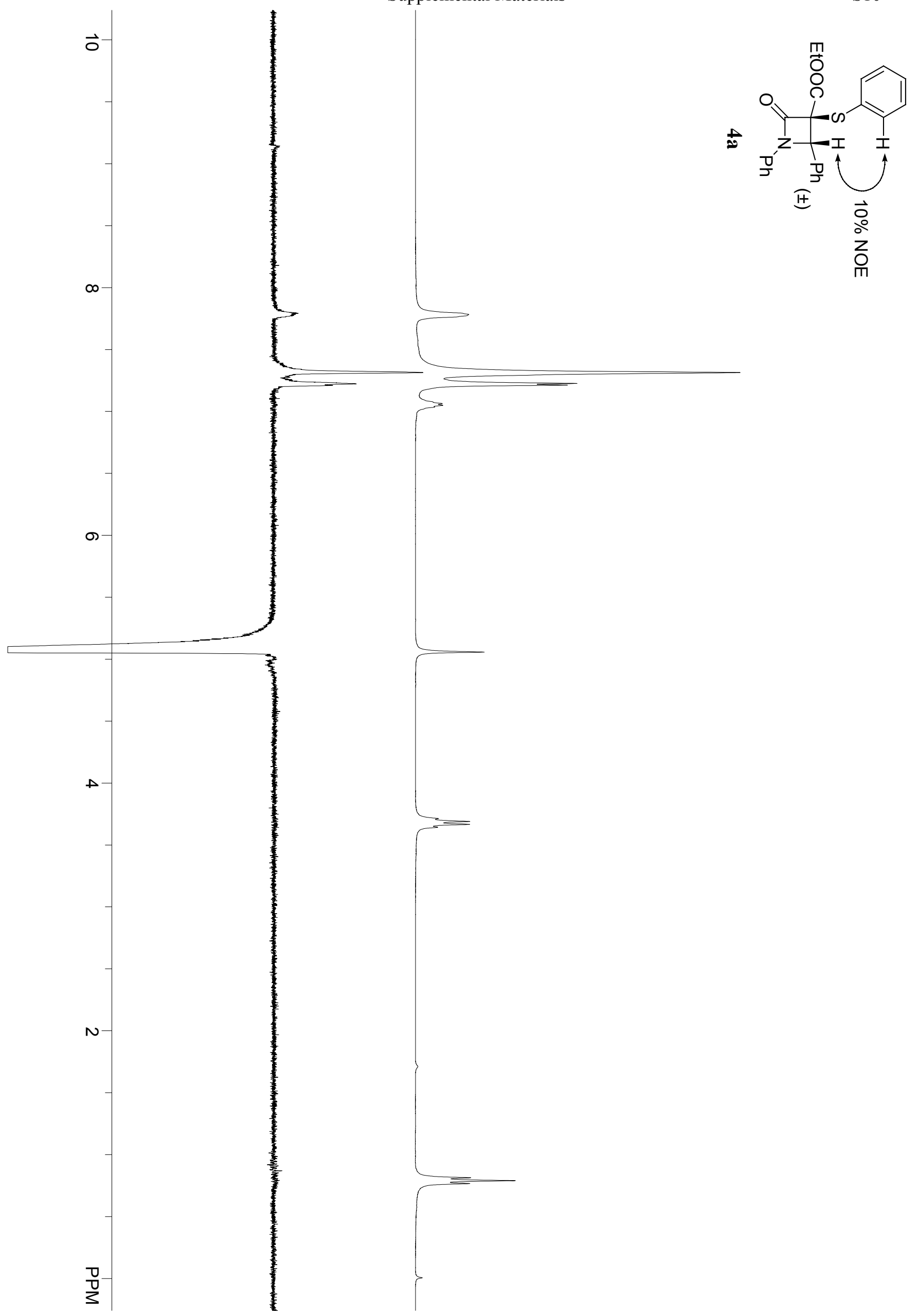




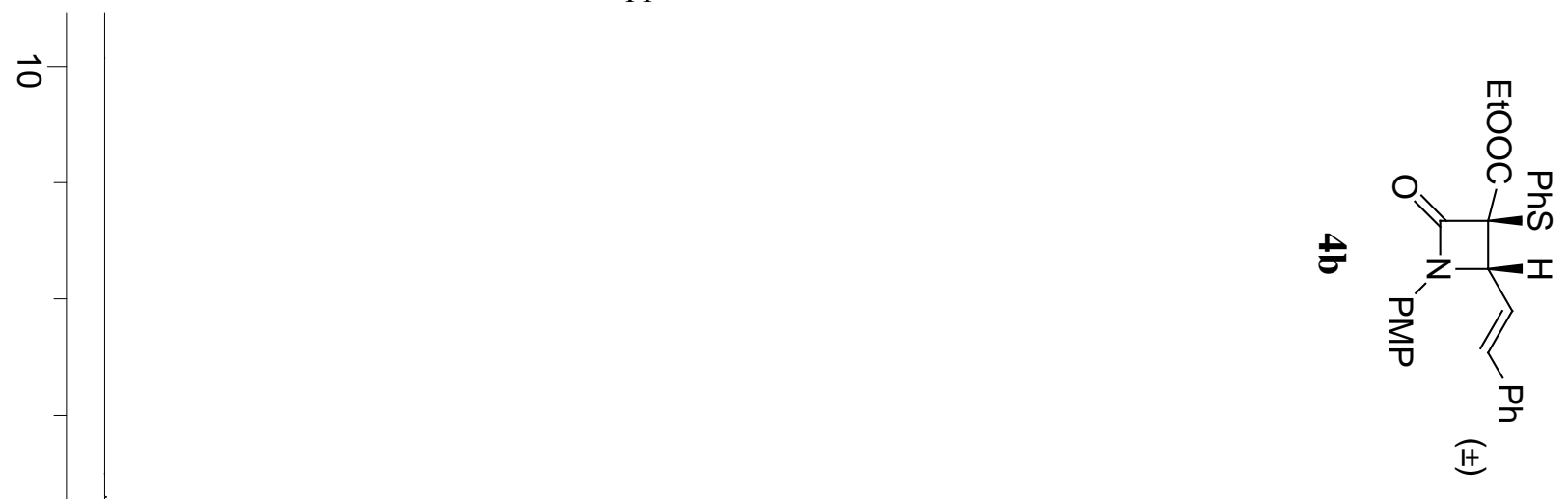

$\infty$

io

$\overrightarrow{0}$

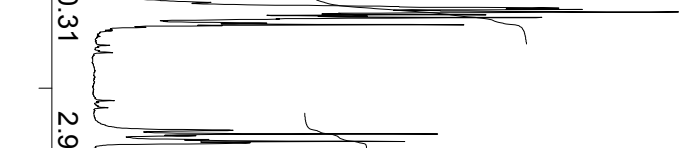

$A$

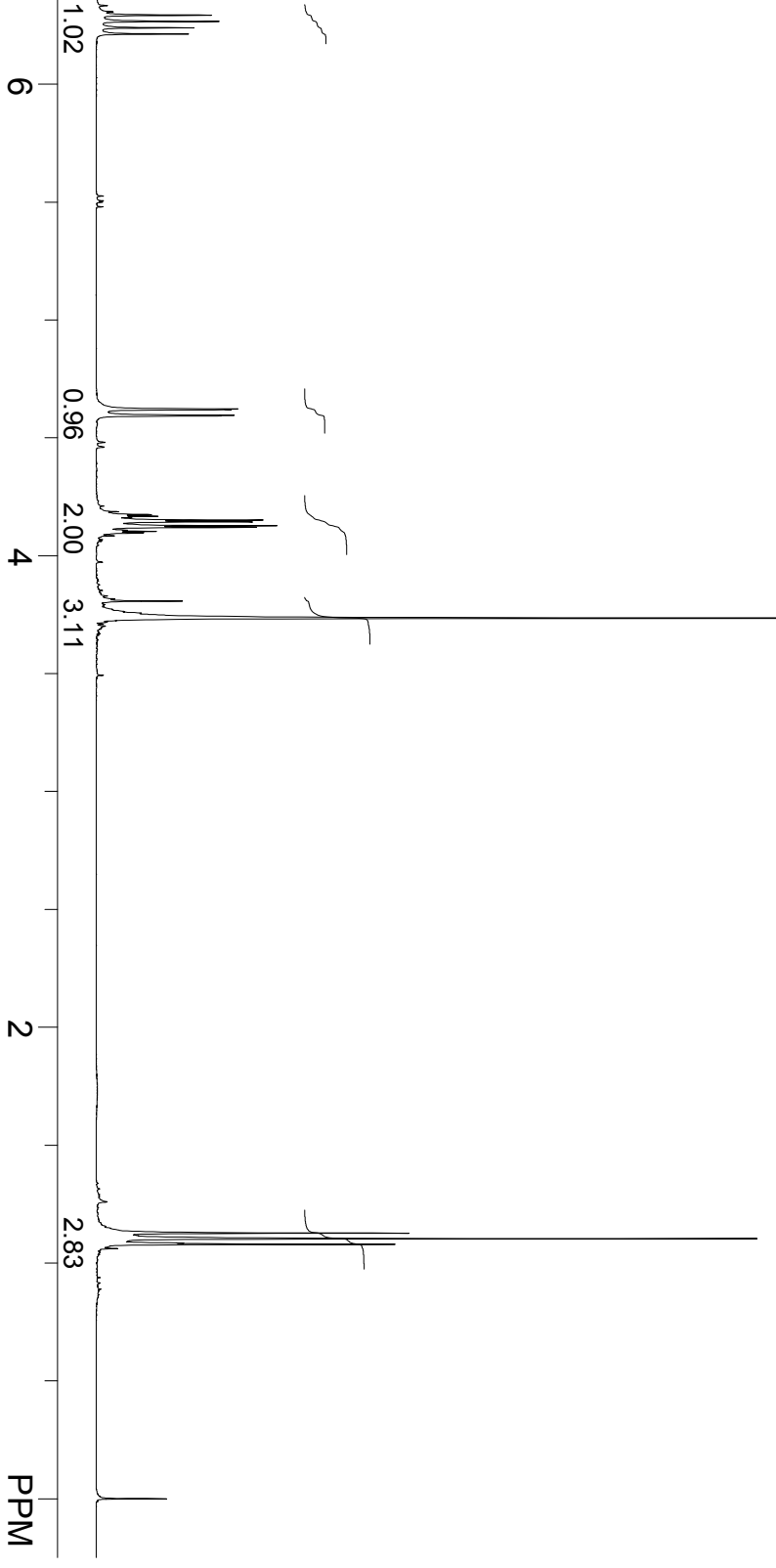




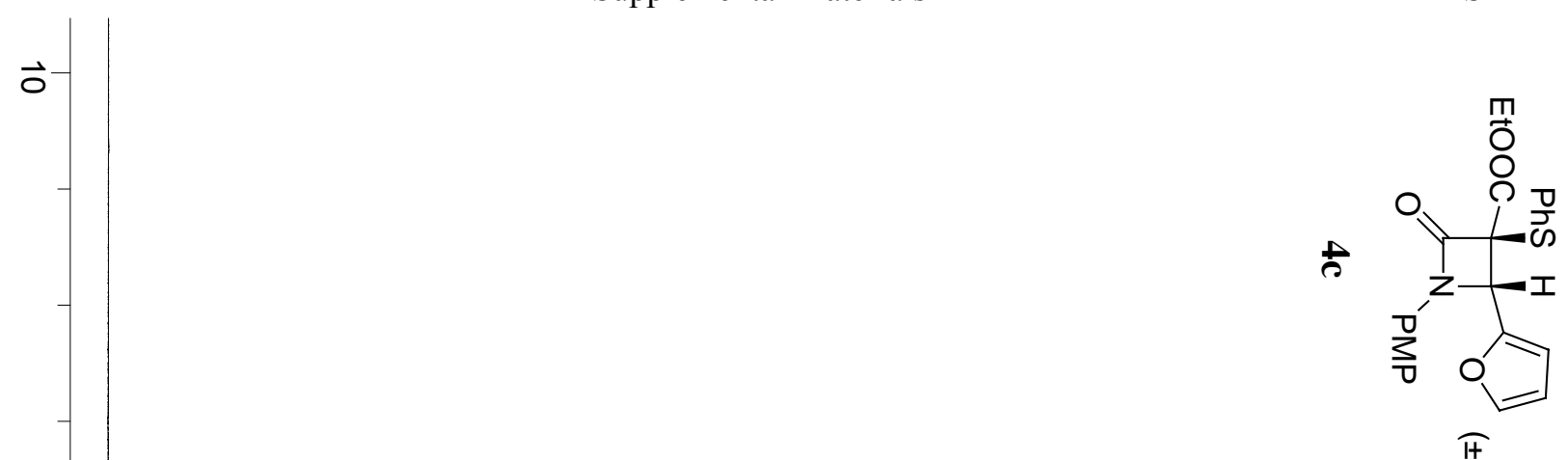

$\infty$
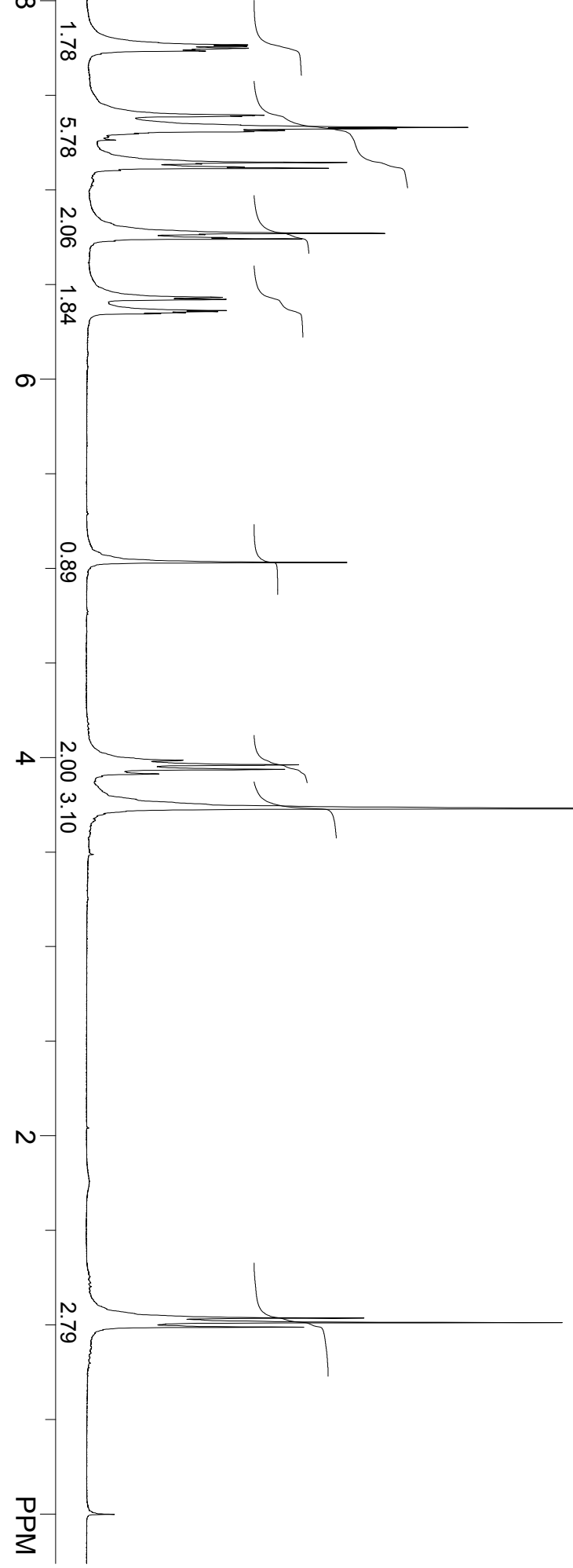


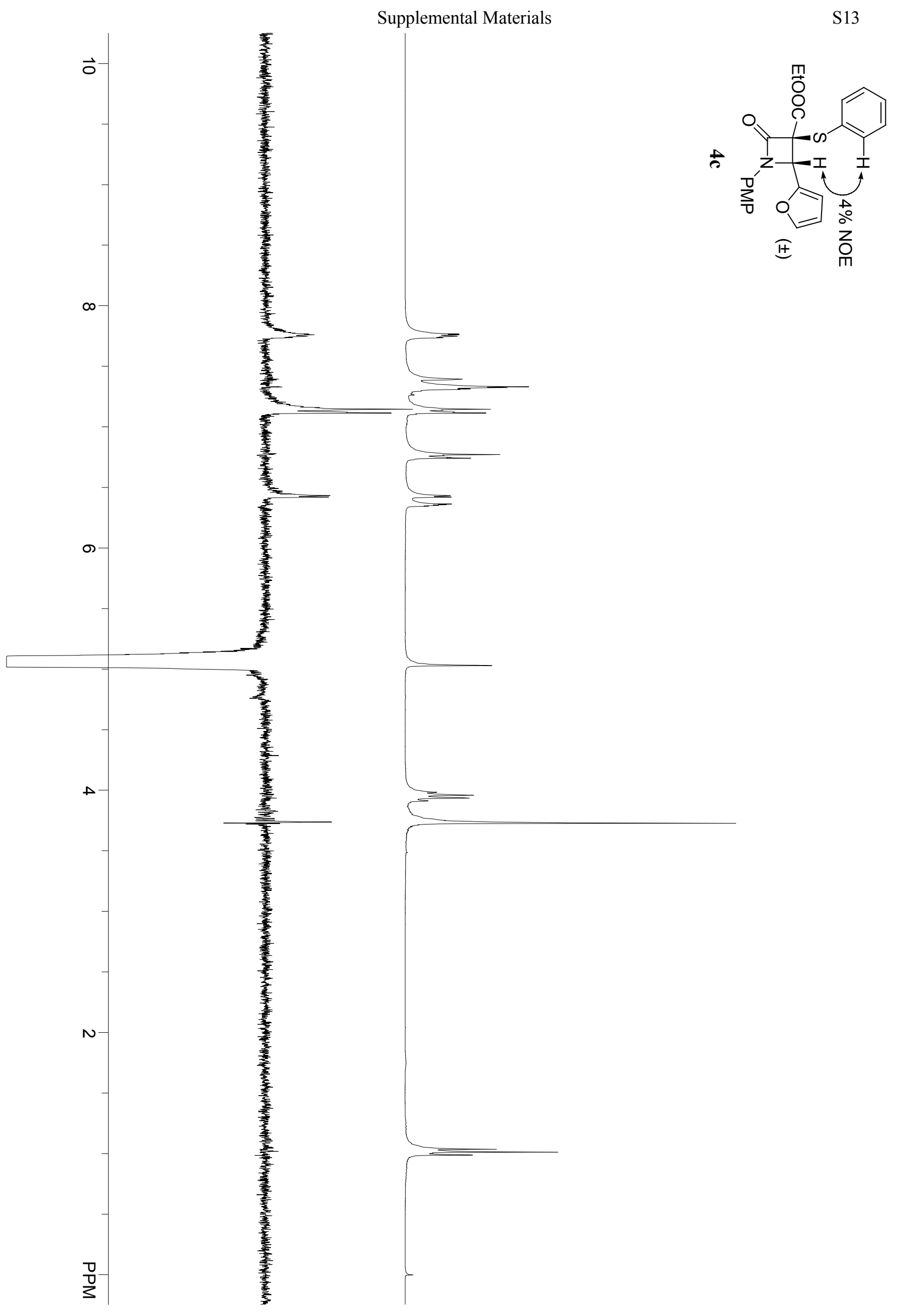


Supplemental Materials

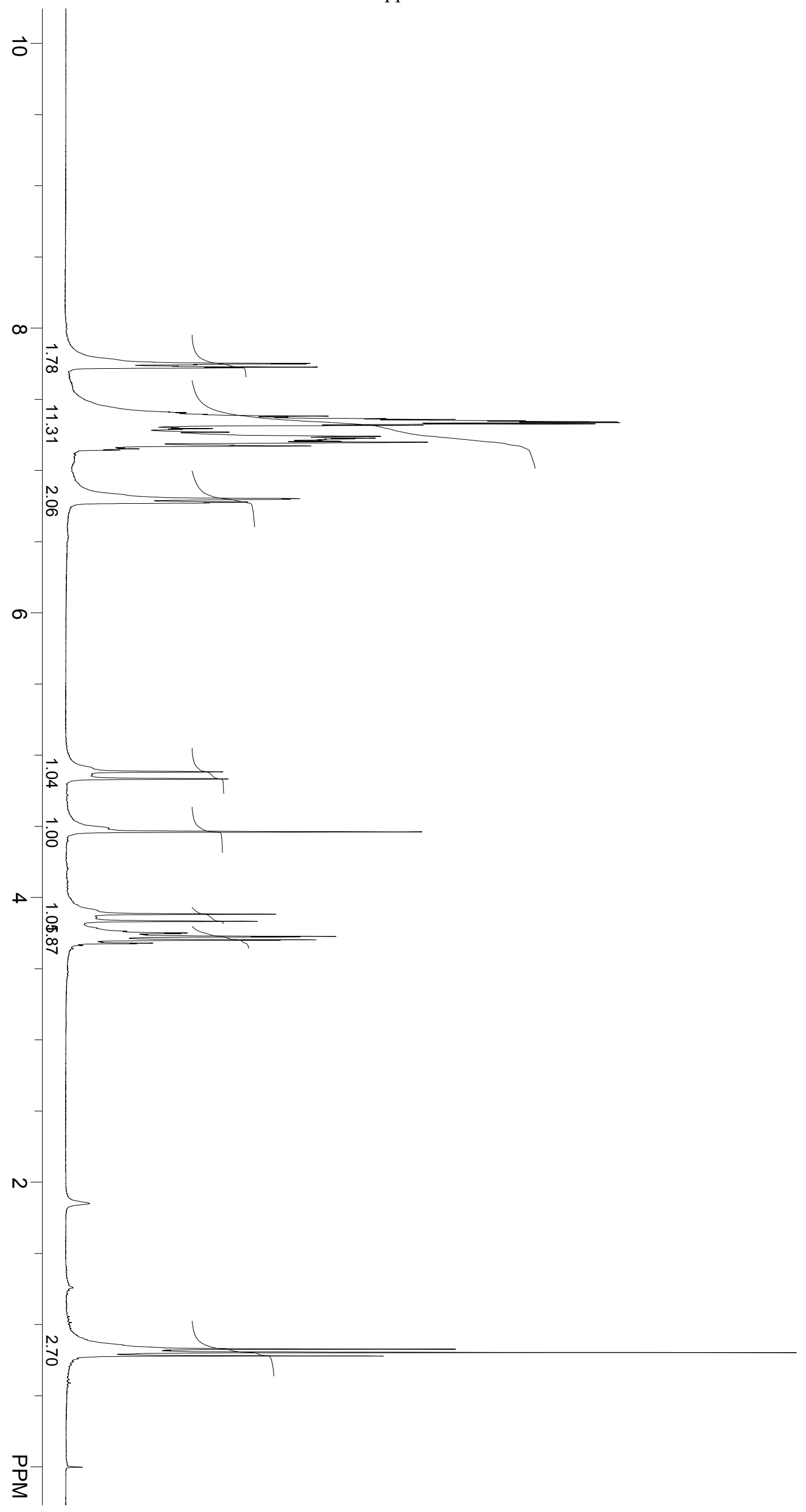

S14

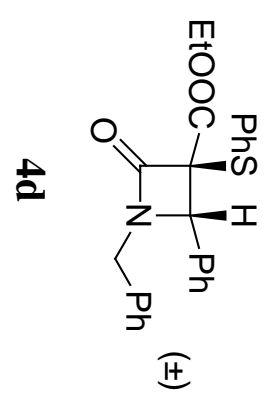




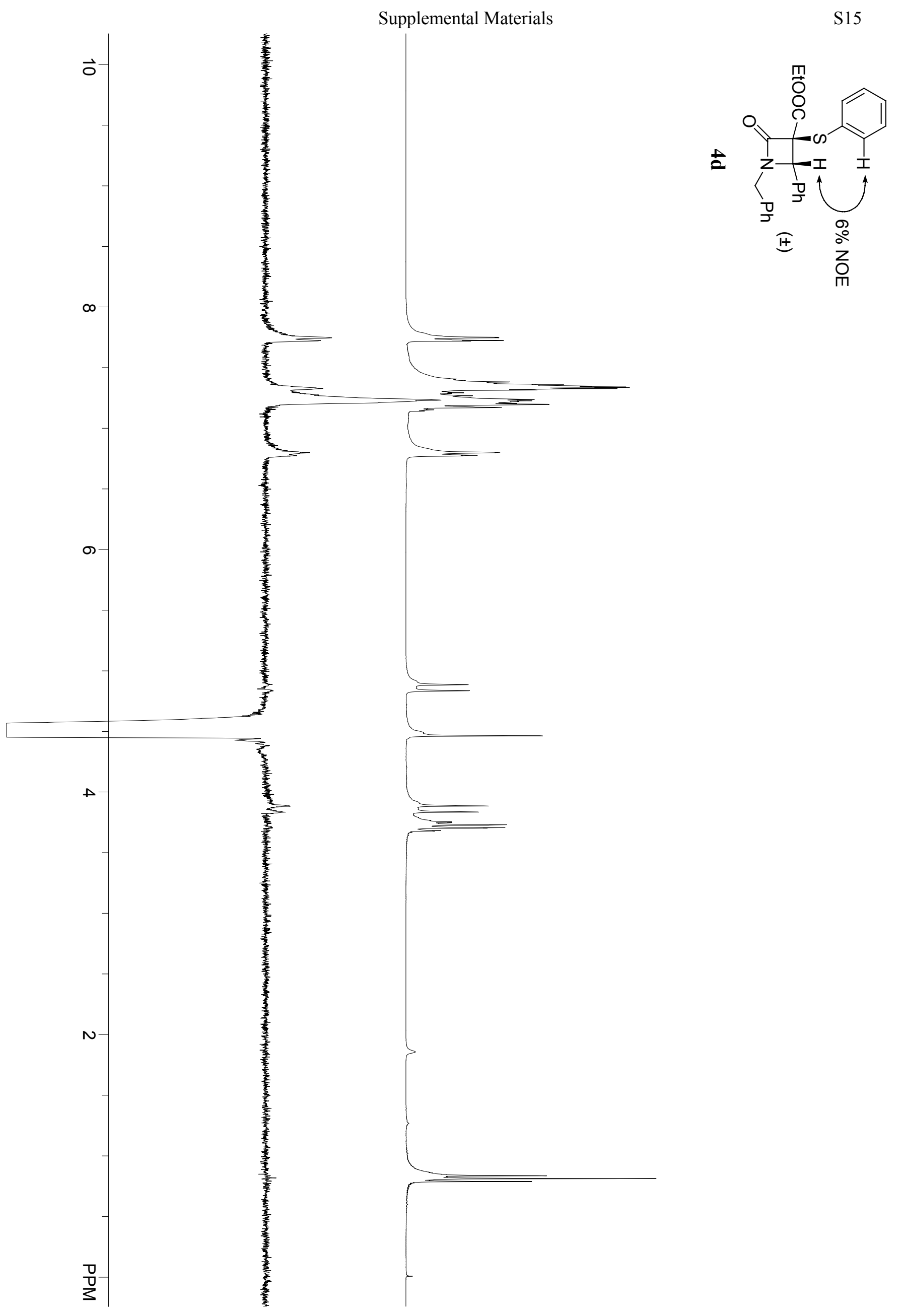




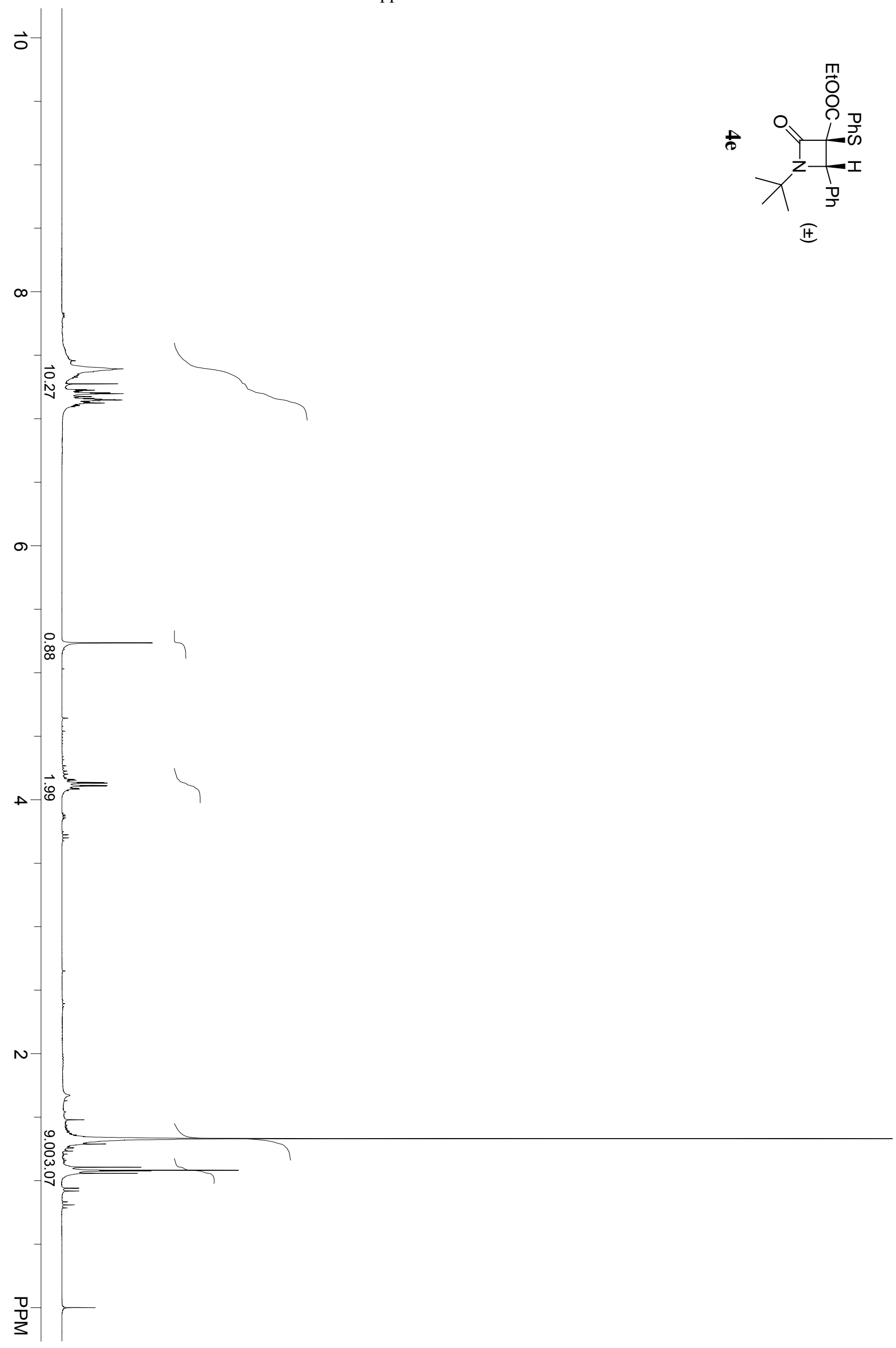




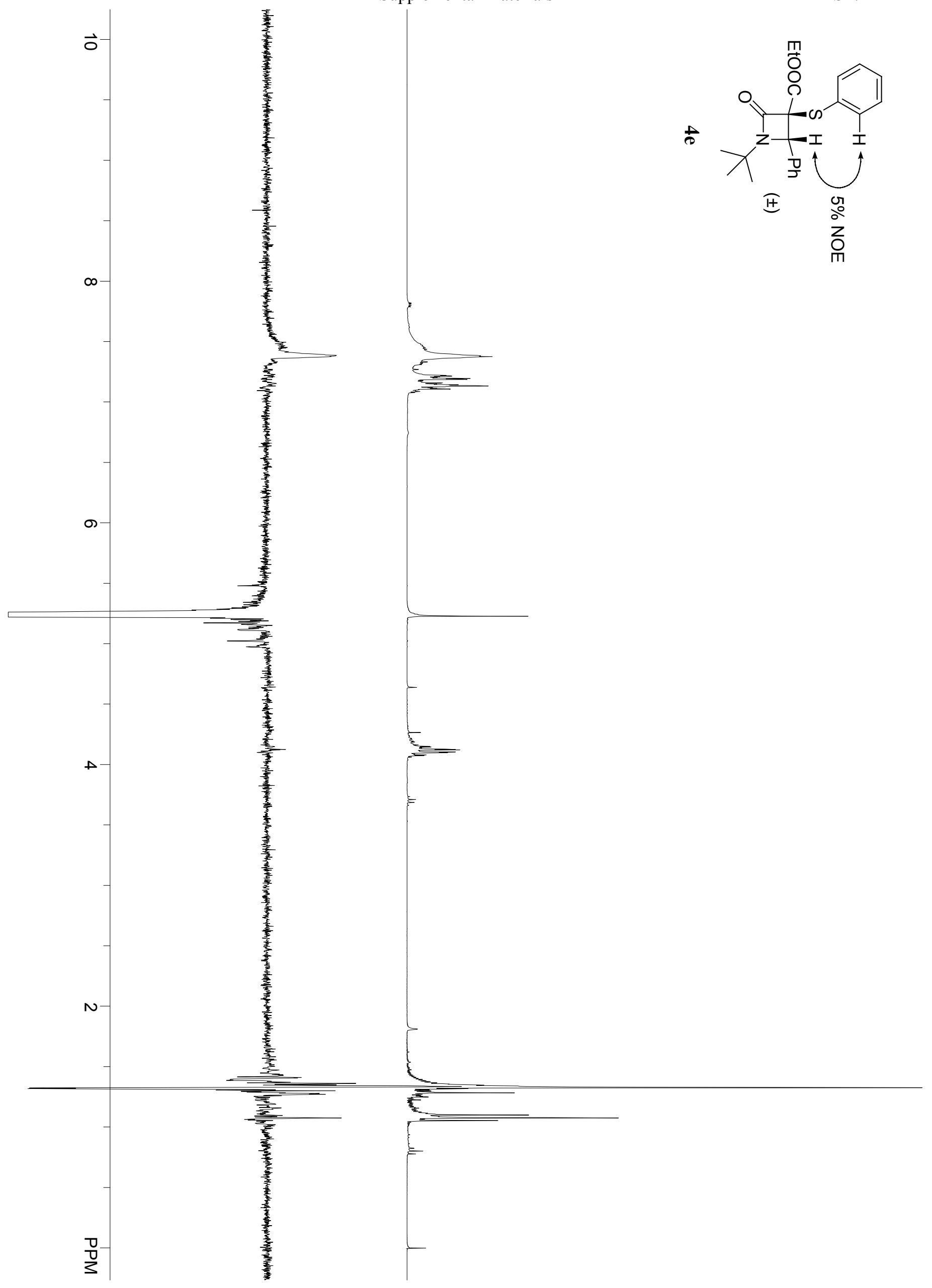




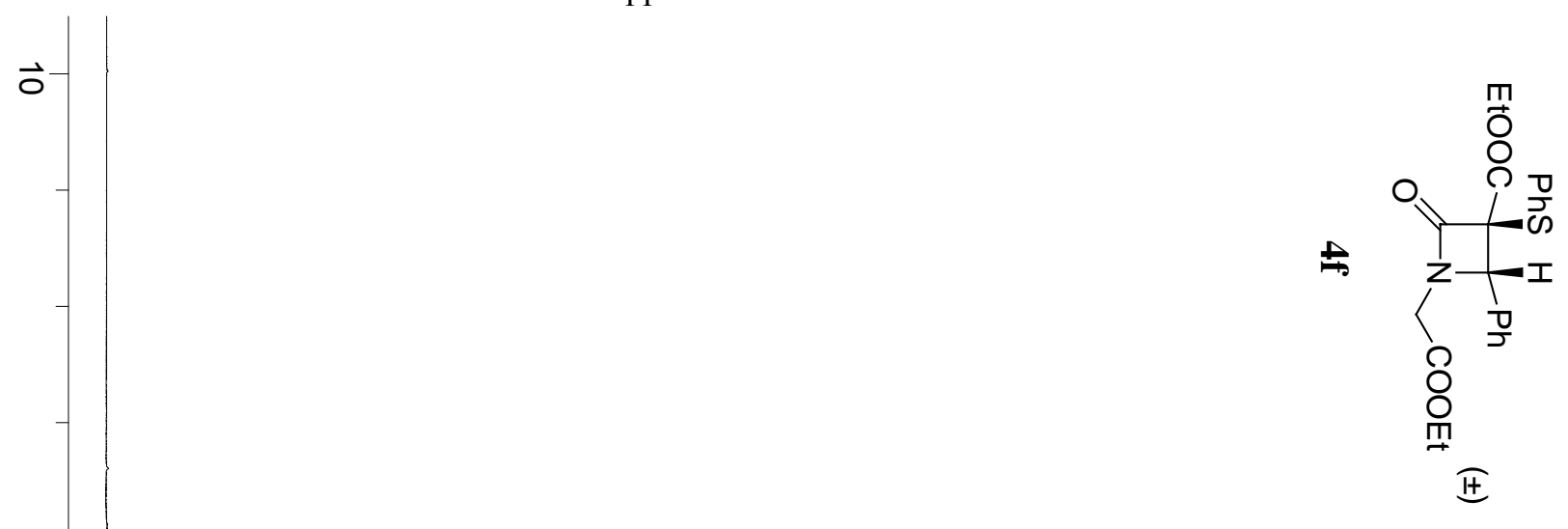

$\infty$

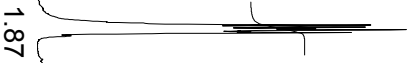

$\infty$

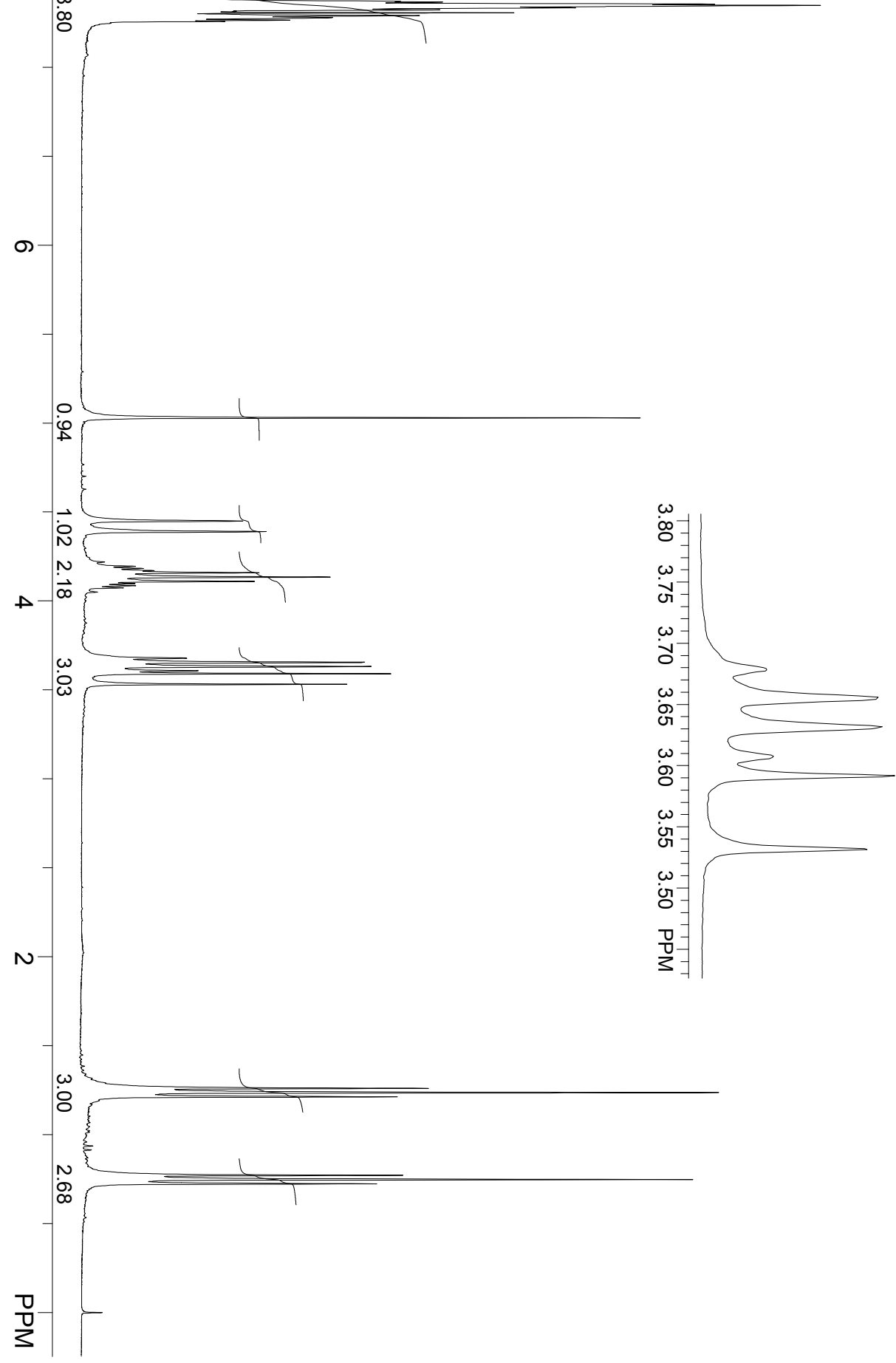




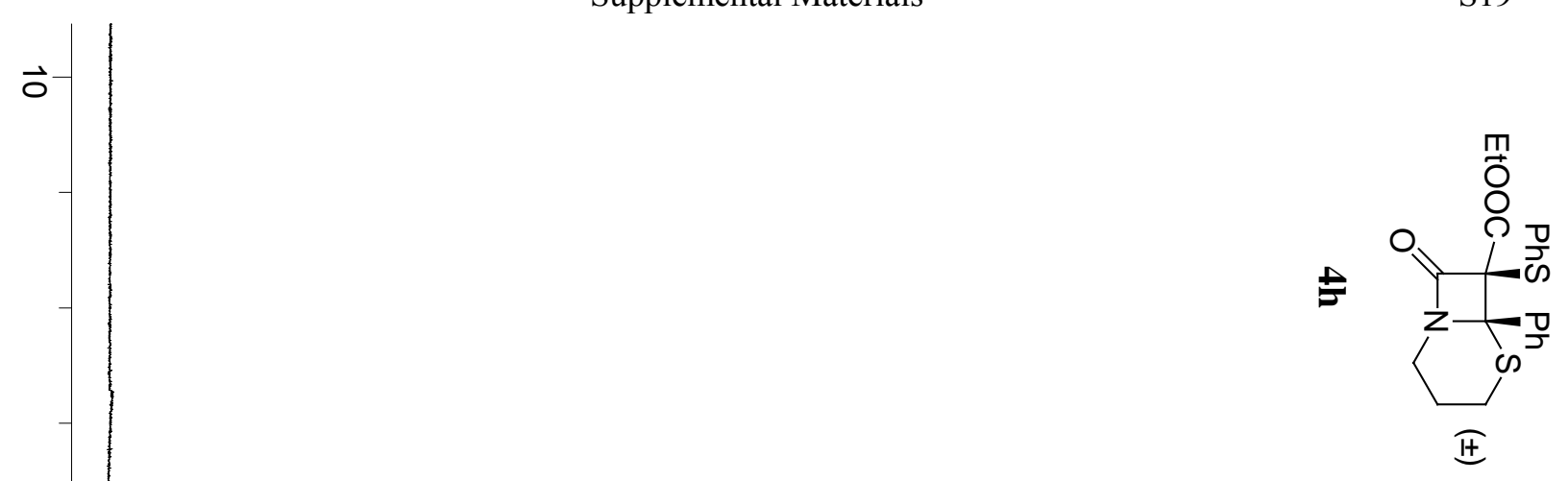

$\infty$

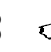

$\sigma$

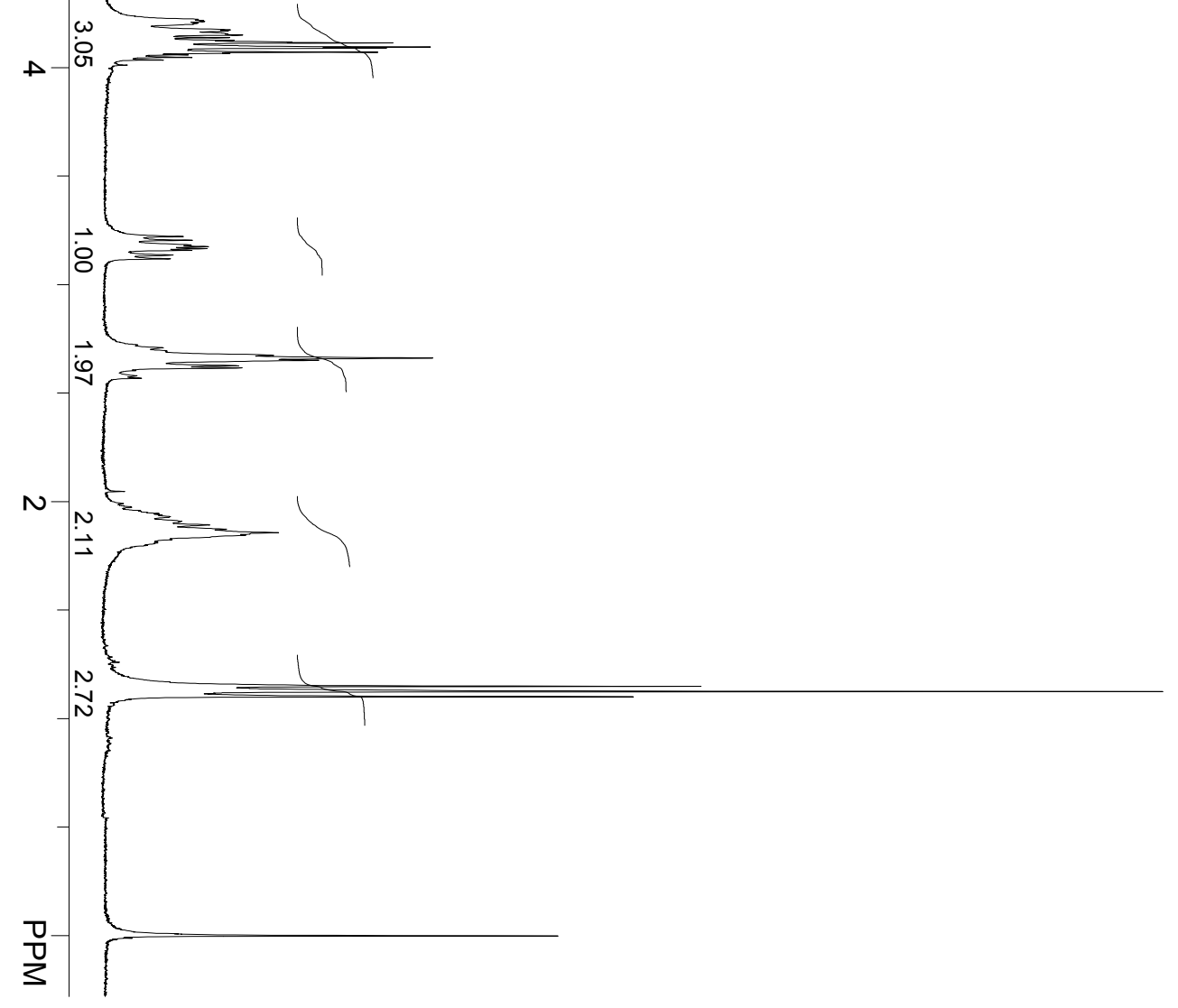




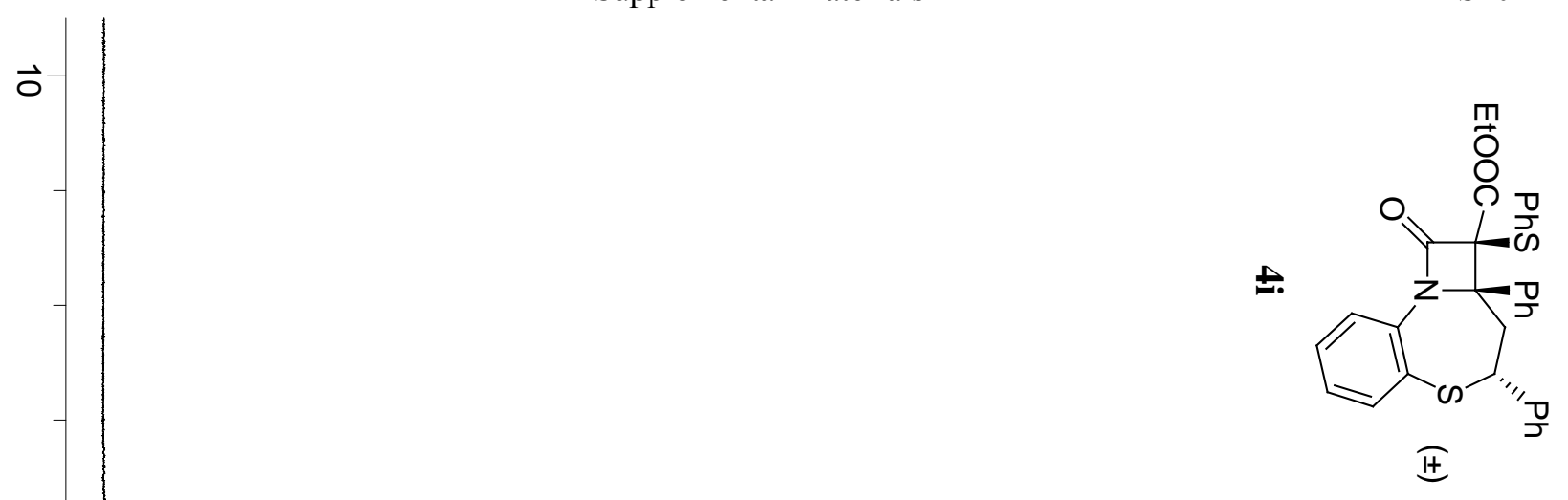

$\infty-$

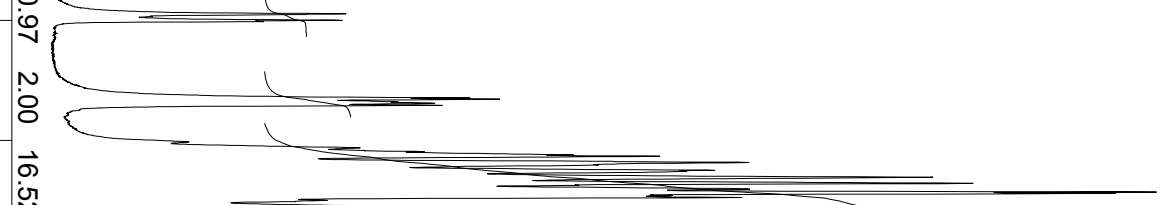

の

N

$\rightarrow$

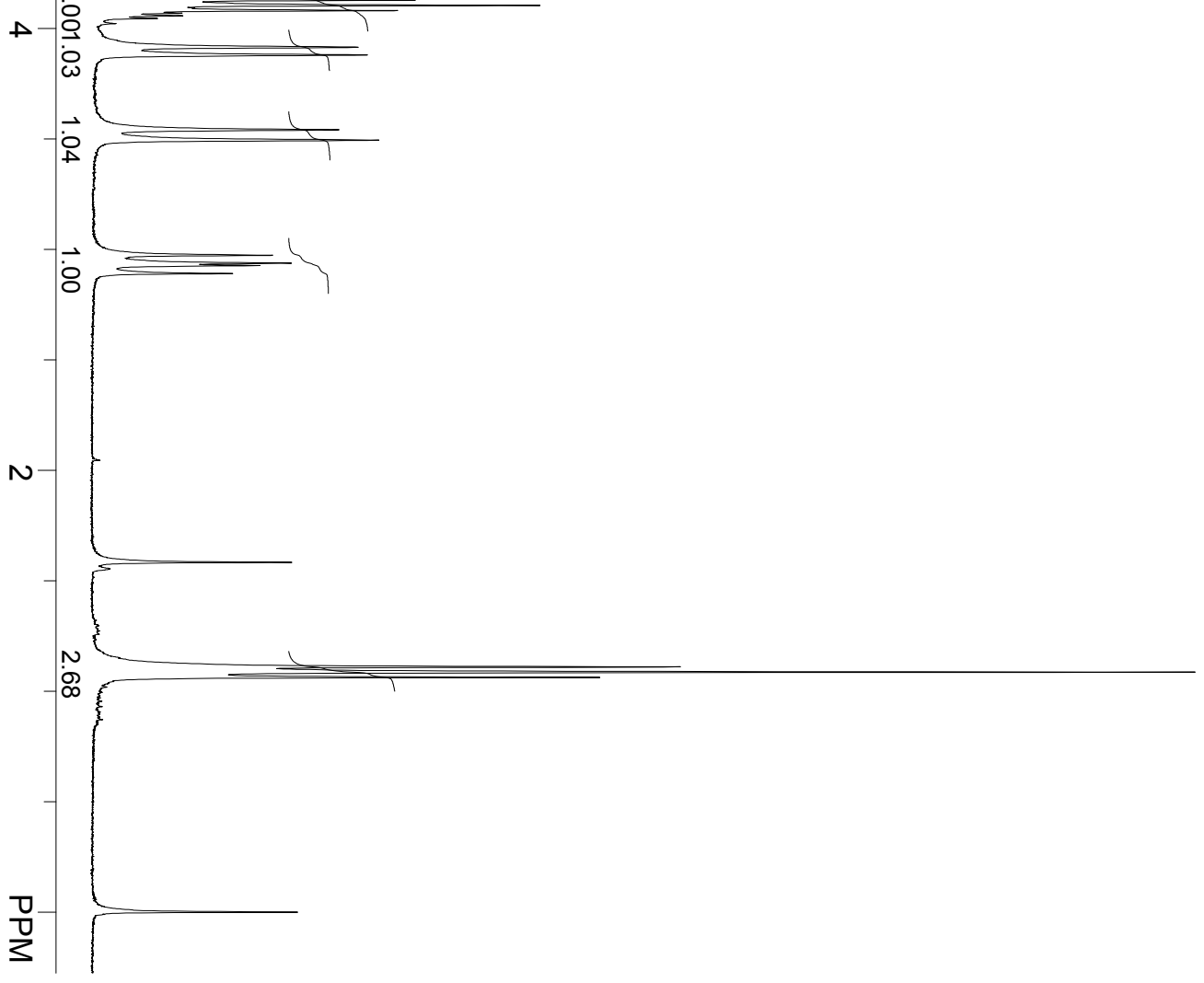




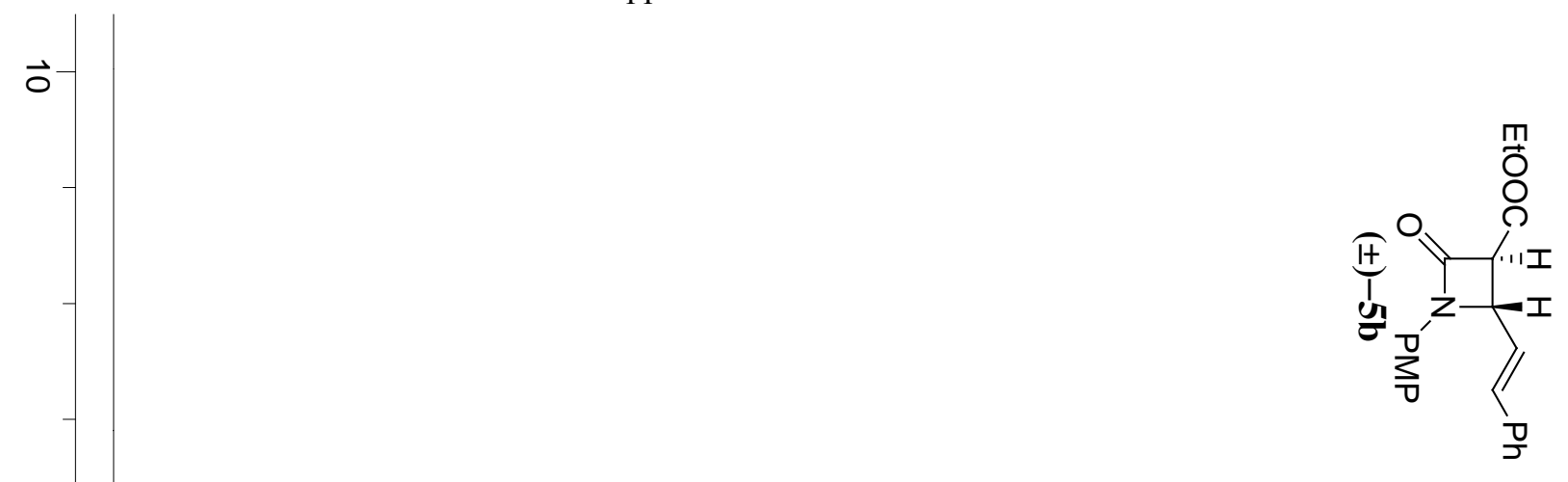

$\infty$

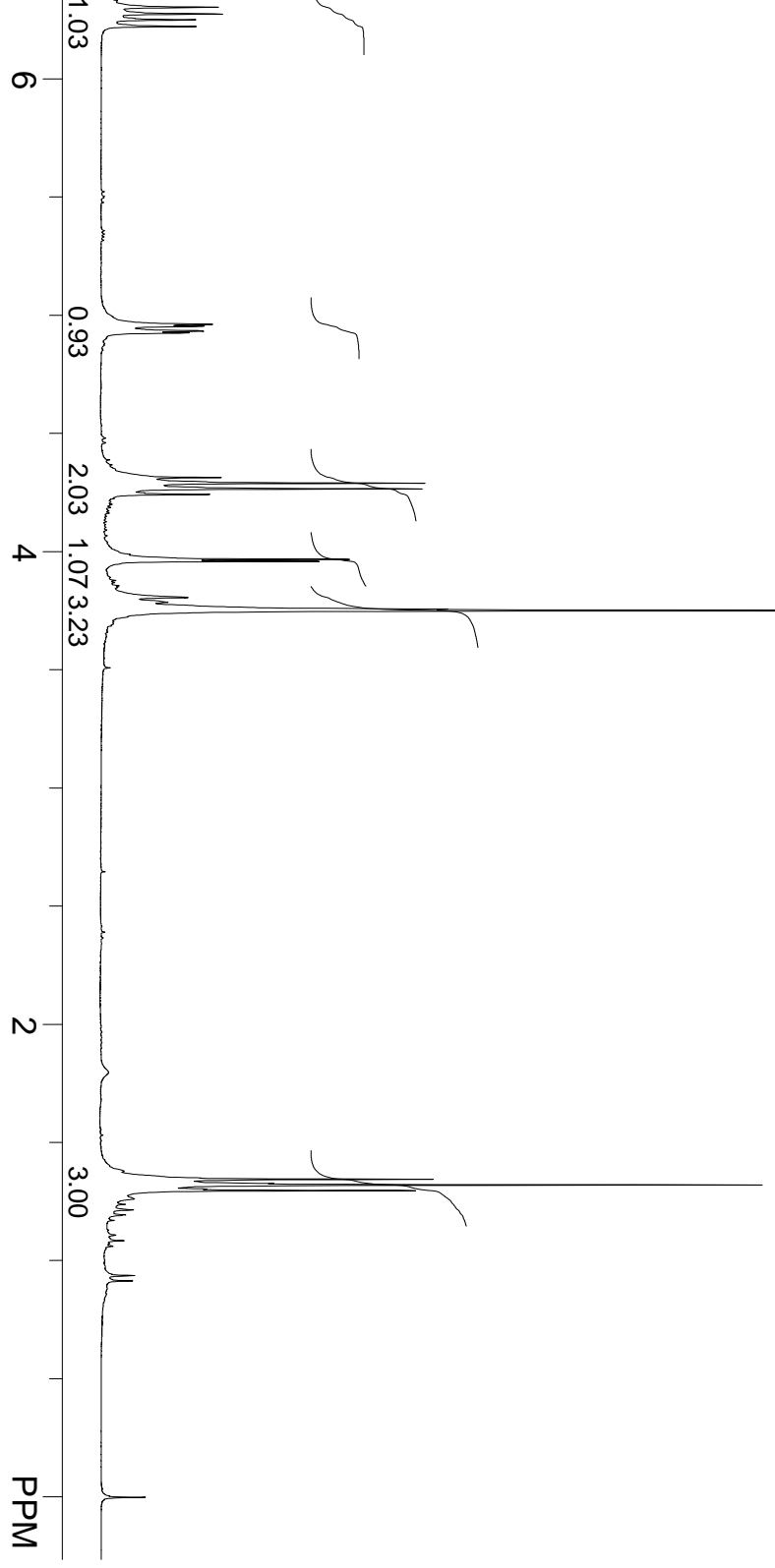




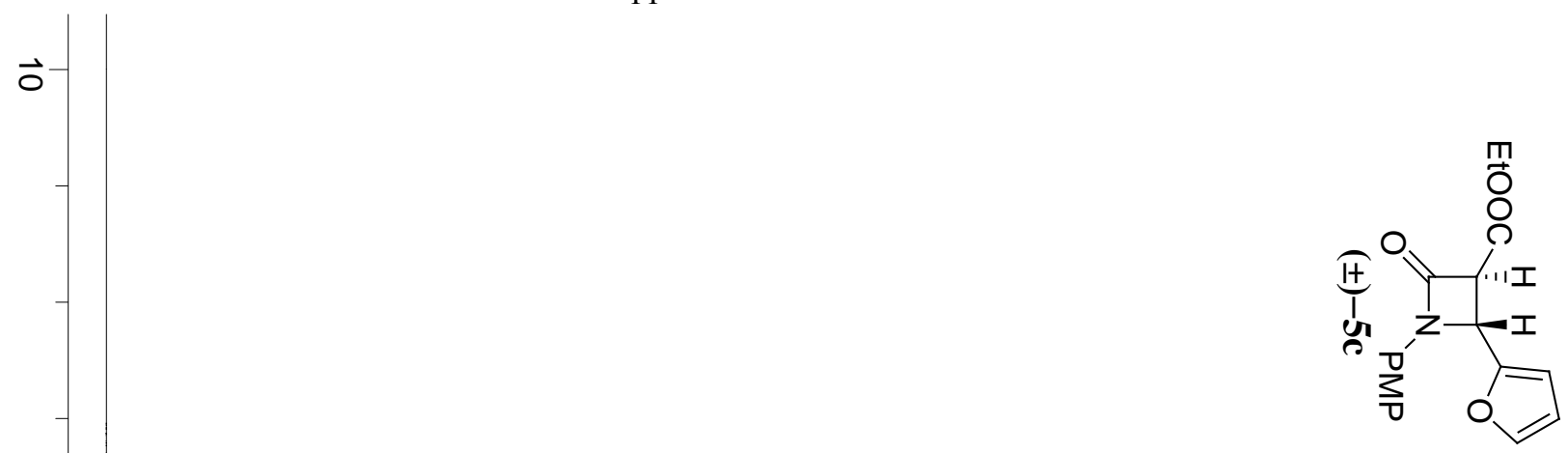

$\infty$
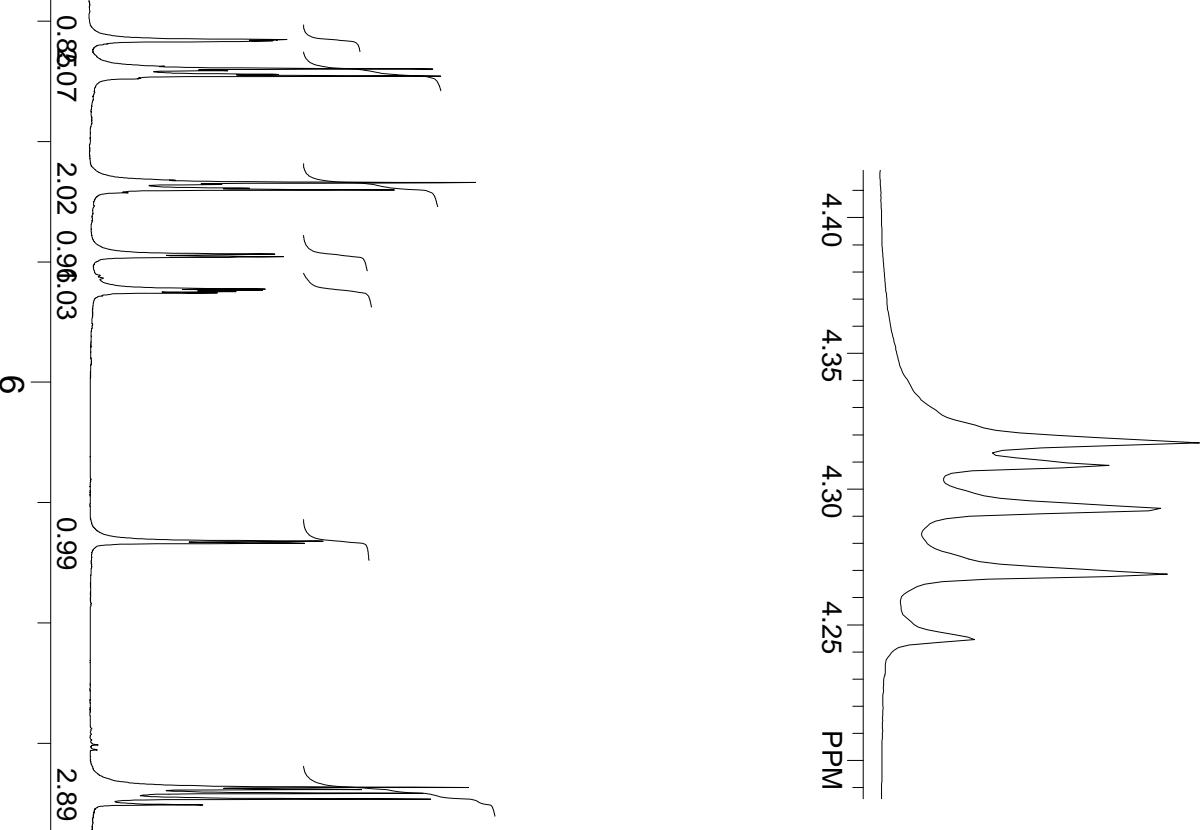

N

$\triangle$

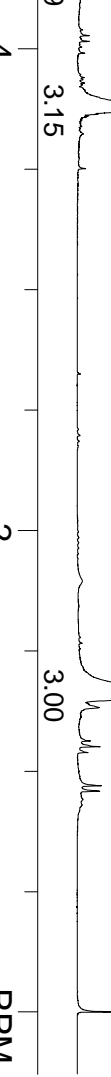




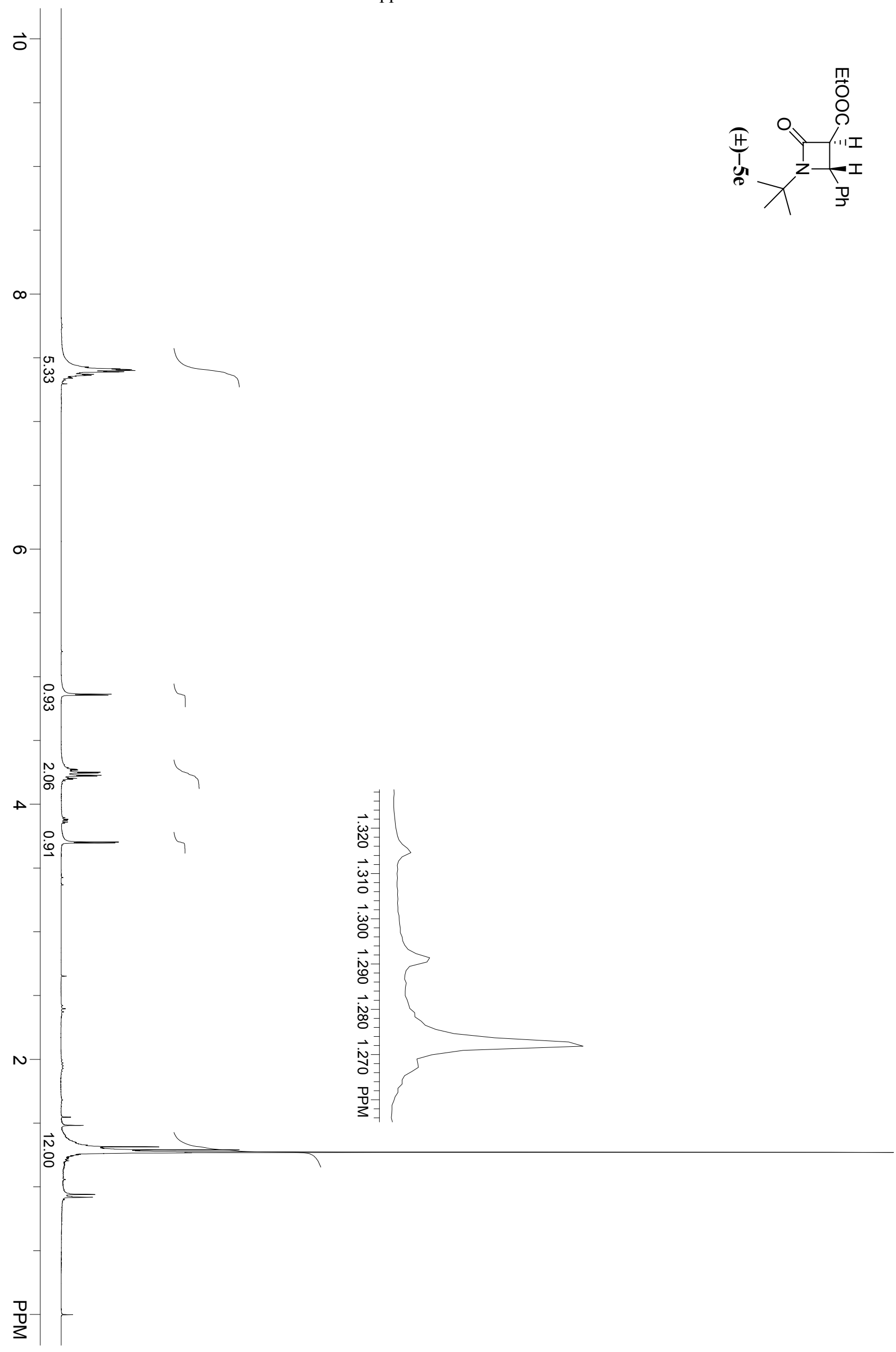




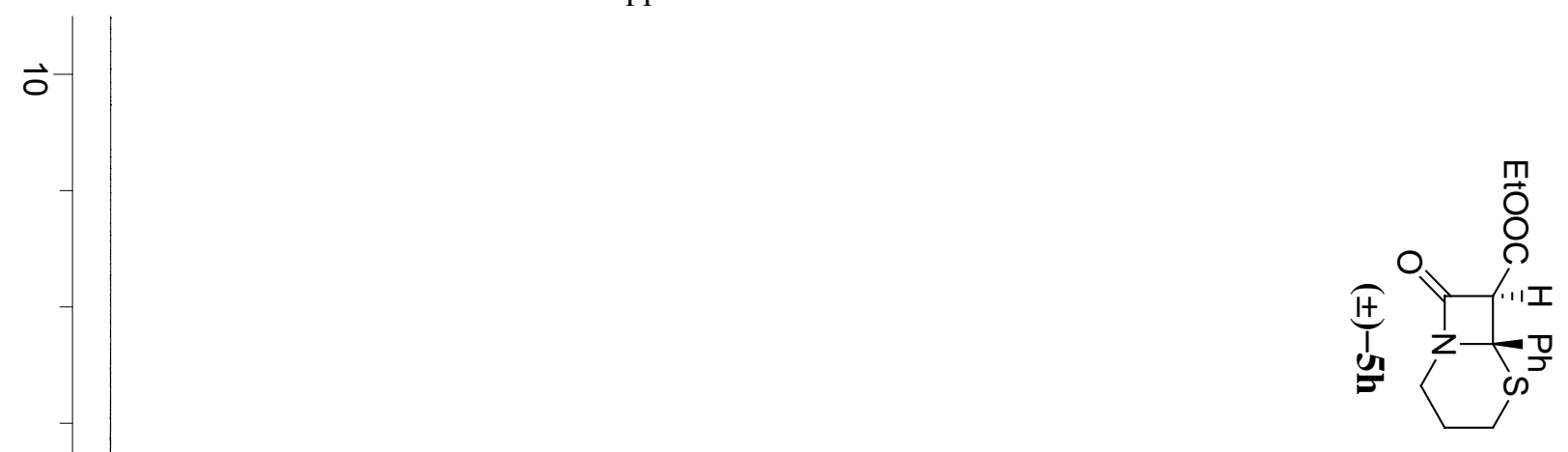

$\infty$

の

$\overrightarrow{\mathrm{o}}$
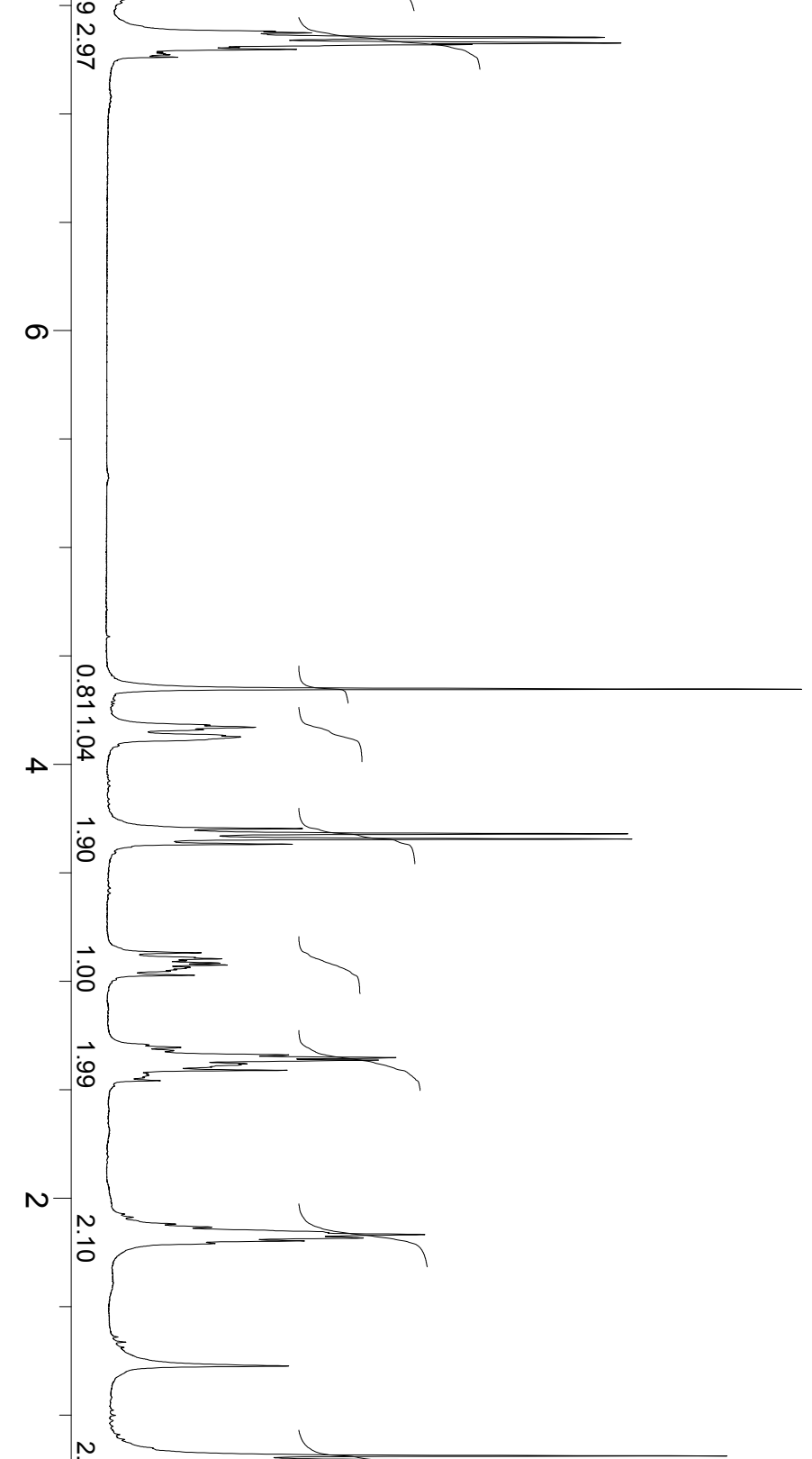

$\underbrace{\infty}$ 
<smiles>CCOC(=O)[C@H]1C(=O)N2c3ccccc3SC(c3ccccc3)C[C@@]12c1ccccc1</smiles>

$( \pm)-5 i$
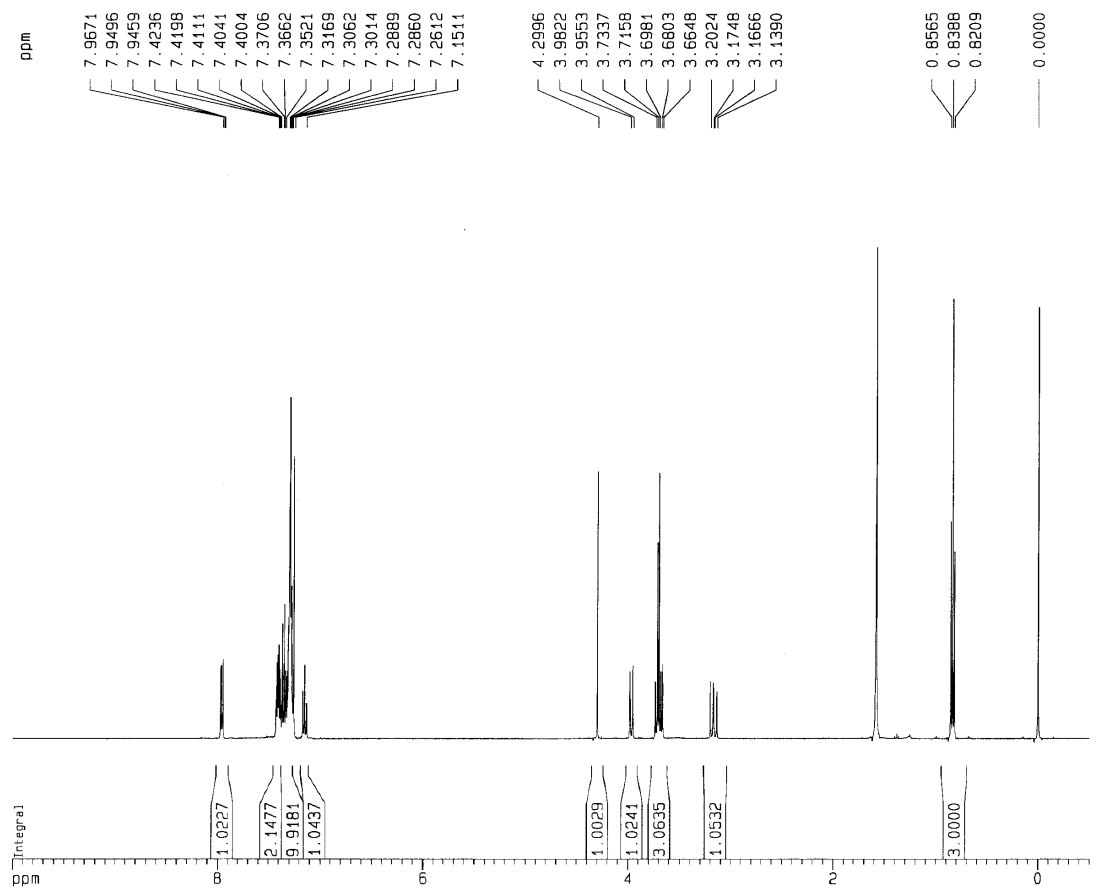

\section{$5 i$ (partially enlarged)}
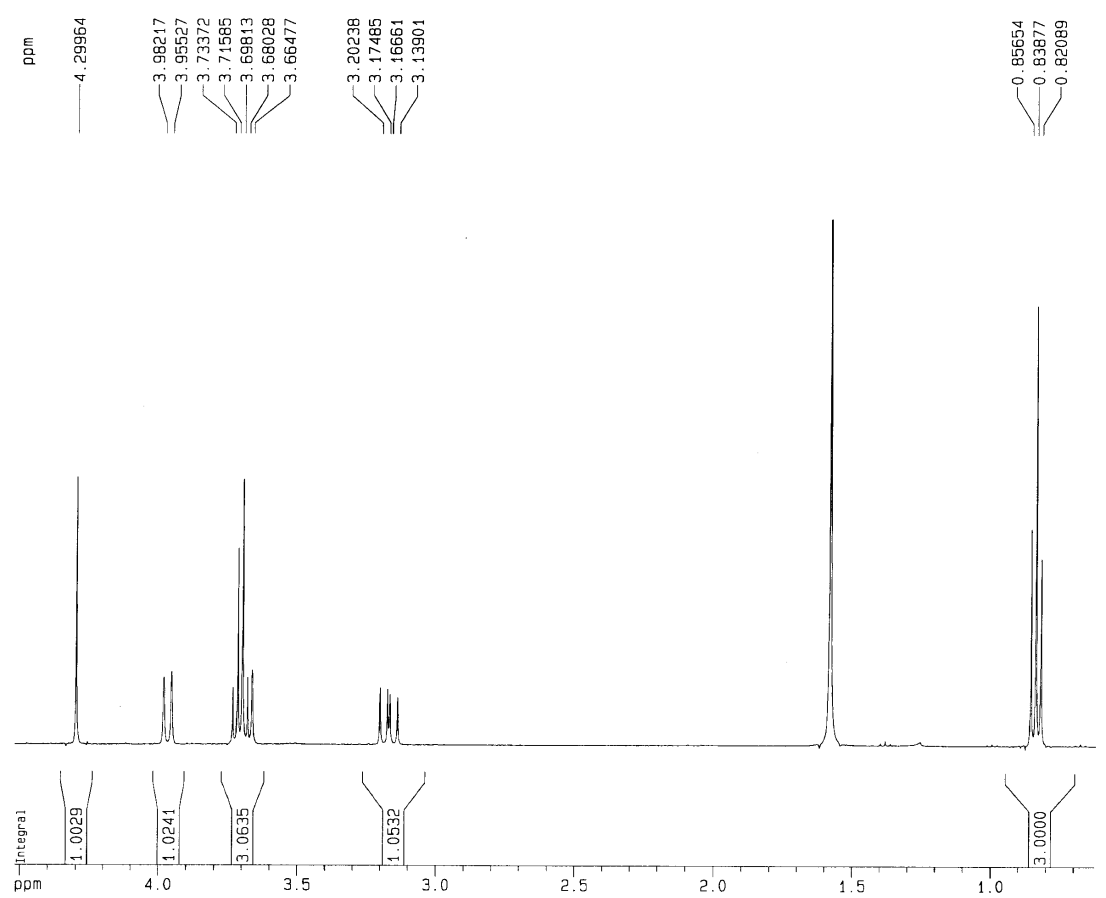
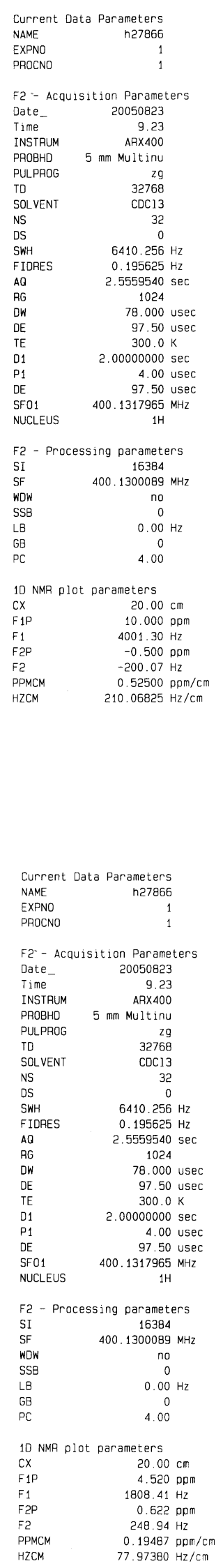

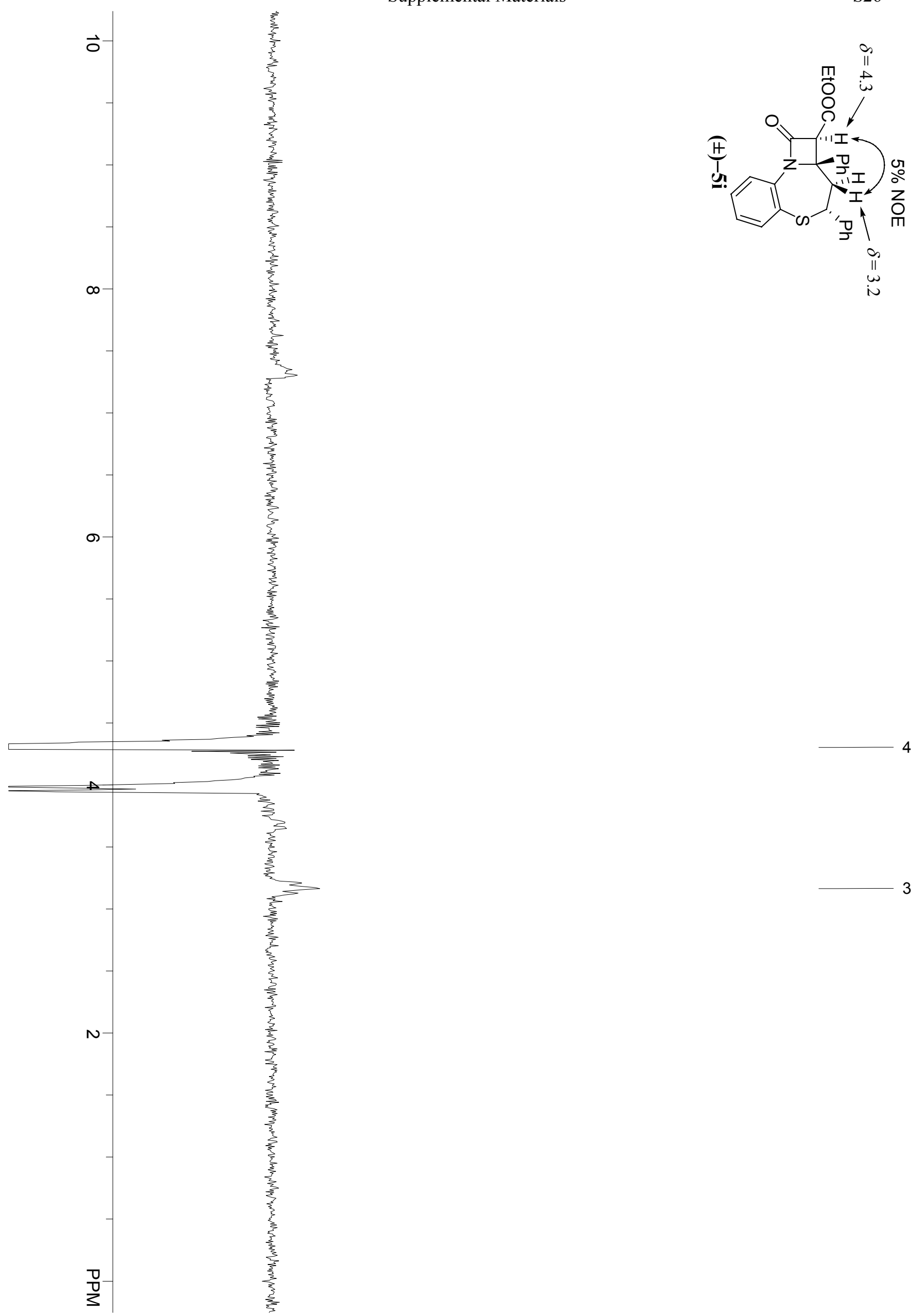


\section{Crystal Structure of Polycyclic $\beta$-Lactam 4i (ORTEP Figure)}<smiles>CCOC(=O)C1(c2ccccc2)CC(c2ccccc2)(c2ccccc2)C(=O)N1c1ccccc1</smiles>
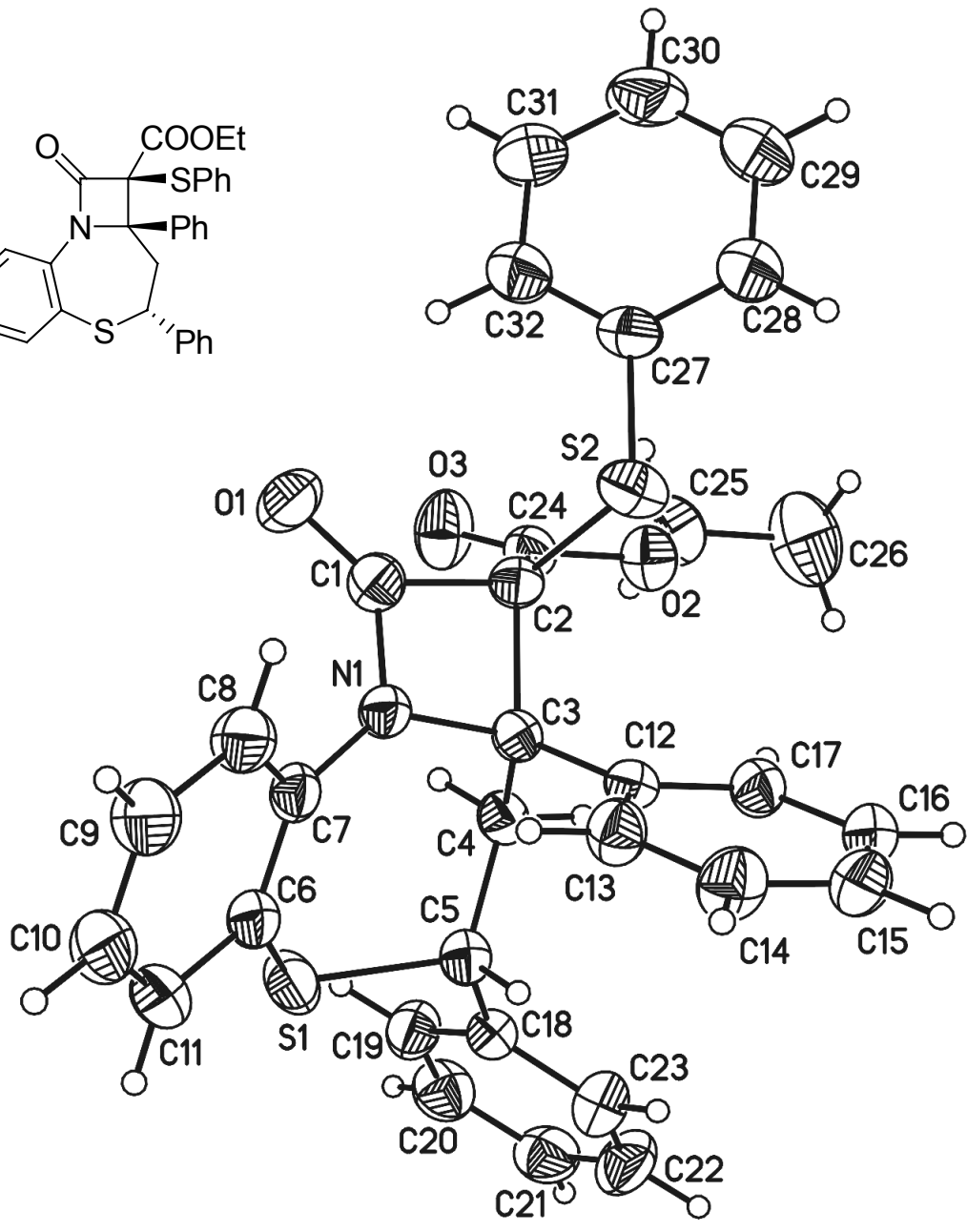

CCDC 283184 contains the supplementary crystallographic data for this paper. These data can be obtained free of charge via www.ccdc.cam.ac.uk/data_request/cif, by emailing data_request@ccdc.cam.ac.uk, or by contacting The Cambridge Crystallographic Data Centre, 12, Union Road, Cambridge CB2 1EZ, UK; fax: +44 1223336033 . 